\title{
Bispectral quantum Knizhnik-Zamolodchikov equations for arbitrary root systems
}

\author{
Michel van Meer
}

Published online: 6 October 2010

(C) The Author(s) 2010. This article is published with open access at Springerlink.com

\begin{abstract}
The bispectral quantum Knizhnik-Zamolodchikov (BqKZ) equation corresponding to the affine Hecke algebra $H$ of type $A_{N-1}$ is a consistent system of $q$-difference equations which in some sense contains two families of Cherednik's quantum affine Knizhnik-Zamolodchikov equations for meromorphic functions with values in principal series representations of $H$. In this paper, we extend this construction of BqKZ to the case where $H$ is the affine Hecke algebra associated with an arbitrary irreducible reduced root system. We construct explicit solutions of $\mathrm{BqKZ}$ and describe its correspondence to a bispectral problem involving Macdonald's $q$-difference operators.
\end{abstract}

Keywords Double affine Hecke algebras · Quantum Knizhnik-Zamolodchikov equations $\cdot$ Bispectral problems

Mathematics Subject Classification (2010) $\quad 81 \mathrm{R} 12 \cdot 20 \mathrm{C} 08$

\section{Introduction}

The bispectral quantum Knizhnik-Zamolodchikov (BqKZ) equations of type $\mathrm{GL}_{N}$ were introduced in [18]. The BqKZ equations make up a consistent system of $q$-difference equations for functions depending on two torus variables $t, \gamma \in T:=$ $(\mathbb{C} \backslash\{0\})^{N}$, such that for fixed $\gamma \in T$, the equations in $t$ form Cherednik's [1] quantum affine Knizhnik-Zamolodchikov (KZ) equations associated with the principal series module $M_{\gamma}$ of the affine Hecke algebra $H$ of type $\mathrm{GL}_{N}$ with central character $\gamma$, while

M. van Meer $(\varangle)$

Korteweg-de Vries Institute for Mathematics, University of Amsterdam,

Science Park 904, 1098 XH Amsterdam, The Netherlands

e-mail: m.vanmeer@uva.nl 
on the other hand, for fixed $t \in T$, the equations in $\gamma$ form another system of quantum affine $\mathrm{KZ}$ equations associated with $M_{t^{-1}}$. The second system is expected to relate to Etingof and Varchenko's system of dynamical $q$-difference equations [8].

Cherednik constructed quantum affine $\mathrm{KZ}$ equations using intertwiners of representations of the affine Hecke algebra. This way different types of quantum affine KZ equations can be associated with different types of affine Hecke algebras and thus to different types of affine root systems. For classical root systems the quantum affine $\mathrm{KZ}$ equations appear as consistency equations for integrable spin chains with different types of boundary conditions (quasi-periodic boundary conditions for $\mathrm{GL}_{N}$ ). In the present paper, we extend the theory of BqKZ and its solutions to arbitrary root systems. Apart from the case of $\mathrm{GL}_{N}$, which was treated in [18], there are three cases to consider in the Macdonald-Cherednik theory, namely the twisted and untwisted reduced affine root systems and the nonreduced affine root system of type $C^{\vee} C$ (see $[14,(1.4 .1)-(1.4 .3)])$. In this paper, we consider the twisted case [14, (1.4.2)]; the untwisted case is expected to allow for a similar treatment. The construction of BqKZ for $C^{\vee} C$ (along the lines of [18]) appeared in a recent preprint by Takeyama [17], so the picture is now rather complete.

Let us explain the ideas involved in a bit more detail. Choose $0<q<1$. Let $W=W_{0} \ltimes P^{\vee}$ be the (extended) affine Weyl group, the semidirect product of the finite Weyl group $W_{0}$ and the coweight lattice $P^{\vee}$, corresponding to some reduced irreducible root system of rank $N$. Consider the complex torus $T:=\operatorname{Hom}_{\mathbb{Z}}\left(P^{\vee}, \mathbb{C}^{\times}\right)$. Transposing the natural action of $W_{0}$ on $P^{\vee}$ gives rise to an action of $W_{0}$ on $T$. For $\lambda \in P^{\vee}$, let $q^{\lambda} \in T$ be defined by

$$
q^{\lambda}(\mu):=q^{\langle\lambda, \mu\rangle}, \quad \mu \in P^{\vee} .
$$

The action of $W_{0}$ on $T$ extends to an action of $W$ on $T$ by letting $\lambda \in P^{\vee}$ act via $t \mapsto q^{\lambda} t$. Let $V$ be a finite-dimensional complex vector space of dimension $\# W_{0}$. The $\mathrm{BqKZ}$ system that we will introduce is a system of $q$-difference equations of the form

$$
C_{(\lambda, \mu)}(t, \gamma) f\left(q^{-\lambda} t, q^{\mu} \gamma\right)=f(t, \gamma), \quad \lambda, \mu \in P^{\vee}
$$

for meromorphic functions $f$ on $T \times T$ with values in $V$. Here, $C_{(\lambda, \mu)}\left(\lambda, \mu \in P^{\vee}\right)$ are $\operatorname{End}(V)$-valued meromorphic functions on $T \times T$, satisfying the following cocycle property

$$
C_{(\lambda+v, \mu+\xi)}(t, \gamma)=C_{(\lambda, \mu)}(t, \gamma) C_{(v, \xi)}\left(q^{-\lambda} t, q^{\mu} \gamma\right), \quad \lambda, \mu, v, \xi \in P^{\vee},
$$

which implies that $\mathrm{BqKZ}$ is a holonomic system of $q$-difference equations.

BqKZ contains, in some sense, two families of Cherednik's quantum affine $\mathrm{KZ}$ equations associated with the principal series representation of $H$. We recall that the quantum affine $\mathrm{KZ}$ equations associated with a finite-dimensional $H$-module $M$ is a consistent system of $q$-difference equations of the form

$$
F_{\lambda}^{M}(t) f\left(q^{-\lambda} t\right)=f(t), \quad \lambda \in P^{\vee},
$$


for meromorphic functions $f$ on $T$ with values in $M$, and where $F_{\lambda}^{M}\left(\lambda \in P^{\vee}\right)$ are $\operatorname{End}(M)$-valued meromorphic functions on $T$ (see Sect. 3.1). Now, the first family of quantum affine $\mathrm{KZ}$ equations inside $\mathrm{BqKZ}$ is parametrized by $\gamma \in T \simeq\{1\} \times T \subset$ $T \times T$. More precisely, if we fix $\gamma=\zeta \in T$, we have

$$
C_{(\lambda, e)}(t, \zeta)=F_{\lambda}^{M_{\zeta}}(t)
$$

where $M_{\zeta}$ is the principal series representation of $H$ with central character $\zeta$, which as a vector space can be identified with $V$ via a $\zeta$-dependent isomorphism. Similarly, interchanging the roles of the torus variables $t$ and $\gamma$, BqKZ contains a second family of quantum affine KZ equations, parametrized by $t \in T$ (related to the affine Hecke algebra module $M_{t^{-1}}$ ).

Let us give an overview of the paper. After the construction of BqKZ, we introduce the principal series representation, needed to express the (asymptotic) values of the connection matrices $C_{(\lambda, \mu)}(t, \gamma)$. These in turn are used to construct an asymptotically free self-dual meromorphic solution $\Phi$ of BqKZ. The set of solutions SOL of BqKZ allows an action of $W_{0}$, and the orbit $W_{0} \Phi$ constitutes a basis of SOL viewed as a vector space over the field of $q$-dilation invariant meromorphic functions on $T \times T$.

For $\mathrm{GL}_{N}$, a correspondence [18, Thm. 6.16] between solutions of BqKZ and solutions of a bispectral problem involving Ruijsenaars' commuting trigonometric $q$-difference operators (also known as Macdonald-Ruijsenaars operators) was derived as a bispectral incarnation of Cherednik's [2, Thm. 4.4] embedding of the solutions of the quantum affine $\mathrm{KZ}$ equations (for $\mathrm{GL}_{N}$ ) into the solutions of the Ruijsenaars eigenvalue problem. The latter has been generalized to an embedding of the solution space of the quantum affine KZ equations for an arbitrary root system into the solution space of a system of $q$-difference equations involving Macdonald's $q$-difference operator (see [9, Thm. 4.6] and [3]). We give the analog of the bispectral correspondence [18, Thm. 6.16] in the setting of arbitrary root systems.

As for $\mathrm{GL}_{N}$, we may apply the correspondence to $\Phi$ to obtain a self-dual Harish-Chandra series solution of the bispectral problem. It is a bispectral analog of (difference) Harish-Chandra series solutions of the spectral problem for Macdonald's $q$-difference operators, which were studied in [7] and [10] for root systems of type $A$ and in [12] for arbitrary root systems. Exploiting the corresponding features of $\Phi$, we obtain new results on the convergence and singularities of the Harish-Chandra series. These results have applications in harmonic analysis on symmetric spaces as well as in relativistic quantum Calogero-Moser systems. Recently, it was shown (see [15]) that for generic $\gamma$, the Harish-Chandra solutions we construct form a basis of the meromorphic solution space of the spectral problem of the Macdonald $q$-difference operators, the quantum integrals of the relativistic quantum Calogero-Moser system. It is expected that this will lead to a $q$-version of Harish-Chandra's $c$-function expansion of the spherical function, an essential step for the $q$-version of harmonic analysis (cf. [16]).

Though the general constructions are more or less the same as for $\mathrm{GL}_{N}$, various technical results require a different approach. This becomes apparent in Sect. 4 when computing the cocycle values, in Sect. 5 determining the asymptotic behavior of the 
$q$-connection matrices and their singularities, and in Sect. 6 finding the leading term of $\Phi$. An important difference with the case of $\mathrm{GL}_{N}$, complicating some of the proofs, is the fact that the affine Weyl group of type $\mathrm{GL}_{N}$ (and the corresponding affine Hecke algebra) allows a rather convenient presentation in terms of the finite Weyl group (respectively finite Hecke algebra) and an affine Dynkin diagram automorphism (see [5, Lemma 1.3.4] or [18, §2.1]), which is lacking for affine Weyl groups (respectively affine Hecke algebras) of arbitrary type. In this paper, we give all the main constructions and provide those proofs that are substantially different from the proofs for $\mathrm{GL}_{N}$.

\subsection{Conventions}

- $\otimes$ always stands for tensor product over $\mathbb{C}$ and $\operatorname{End}(M)$, for a module $M$ over $\mathbb{C}$, stands for $\mathbb{C}$-linear endomorphisms.

$-\mathbb{N}=\{1,2, \ldots\}$.

- For a module $M$ over a commutative ring $R$ and a ring extension $R \subset S$, we write $M^{S}=S \otimes_{R} M$.

- For $a, r \in \mathbb{R}$ with $a>0$, we choose $a^{r}$ to be the positive real branch of the power function.

\section{Notations}

\subsection{Root data}

Let $(V,\langle\rangle$,$) be a real Euclidean space of dimension N>0$. Let $\widehat{V}$ be the space of affine linear real functions on $V$. Consider the 1 -dimensional vector space $\mathbb{R} c$. There is a natural isomorphism of real vector spaces $V \oplus \mathbb{R} c \simeq \widehat{V}$ via $v+r c \mapsto(u \mapsto\langle v, u\rangle+r)$ for $u, v \in V$ and $r \in \mathbb{R}$. We will use this isomorphism to identify $\widehat{V}$ and $V \oplus \mathbb{R} c$, thus regarding $c \in \widehat{V}$ as the constant function equal to 1 .

The map $D: \widehat{V} \rightarrow V$ defined by $D(v+r c)=v(v \in \mathbb{R}, r \in \mathbb{R})$ is called the gradient map. We extend the inner product $\langle$,$\rangle to a positive semi-definite bilinear$ form on $\widehat{V}$ by

$$
\langle f, g\rangle:=\langle D f, D g\rangle
$$

for $f, g \in \widehat{V}$. For $f \in \widehat{V}$ with $D f \neq 0$, we set $f^{\vee}:=2 f /\langle f, f\rangle \in \widehat{V}$.

Let $R \subset V$ be a reduced irreducible finite root system in $V$ and assume that the scalar product is normalized such that long roots have squared length 2 . The Weyl group $W_{0} \subset O(V)$ associated with $R$ is the group generated by the orthogonal reflections $s_{\alpha}$ in the hyperplanes $\alpha^{\perp}(\alpha \in R)$. Explicitly, we have

$$
s_{\alpha}(v)=v-\langle v, \alpha\rangle \alpha^{\vee}
$$


for $\alpha \in R, v \in V$. Fix a basis of simple roots $\left\{\alpha_{1}, \ldots, \alpha_{N}\right\}$ of $R$. Write $R_{+}$for the set of positive roots, $R_{-}:=-R_{+}$for the set of negative roots, and $\phi$ for the highest root with respect to this basis. Note that $\phi \in R_{+}$is a long root (and so $\phi^{\vee}=\phi$ ).

We use the standard notations for the (co)root and (co)weight lattices, that is,

$$
\begin{aligned}
Q & :=\mathbb{Z} \text {-span of } R, \\
Q^{\vee} & :=\mathbb{Z} \text {-span of } R^{\vee}, \\
P & :=\left\{\lambda \in V \mid\left\langle\lambda, \alpha^{\vee}\right\rangle \in \mathbb{Z}, \forall \alpha \in R\right\}, \\
P^{\vee} & :=\{\mu \in V \mid\langle\mu, \alpha\rangle \in \mathbb{Z}, \forall \alpha \in R\} .
\end{aligned}
$$

Note that $Q \subseteq P$ and $Q^{\vee} \subseteq P^{\vee}$. Furthermore, since $\|\alpha\|^{2}=2$ for $\alpha \in R$ a long root and thus $\|\alpha\|^{2} \in\{1,2 / 3\}$ for $\alpha \in R$ short, we have $\alpha^{\vee}=\frac{2}{\|\alpha\|} \alpha \in\{\alpha, 2 \alpha, 3 \alpha\} \subset Q$ for any $\alpha \in R$. Hence, $Q^{\vee} \subseteq Q$ and therefore also $P^{\vee} \subseteq P$.

Let $L \subset V$ be any $W_{0}$-invariant lattice. The canonical action of $W_{0}$ on $V$ extends to a faithful action of the semi-direct product group $W_{L}:=W_{0} \ltimes L$ on $V$ such that elements of $L$ act as translations. If we want to stress that we view $\lambda \in L$ as an element of $W_{L}$, we write $\mathrm{t}(\lambda)$. In this notation, $L \subset W_{L}$ acts on $V$ by

$$
\mathrm{t}(\lambda) v=v+\lambda
$$

for $\lambda \in L$ and $v \in V$. Transposing the action of $W_{L}$ on $V$ gives an action of $W_{L}$ on $\widehat{V}$. It is given by

$$
\begin{aligned}
w(v+r c) & =w(v)+r c, \quad w \in W_{0}, \\
\mathrm{t}(\lambda)(v+r c) & =v+(r-\langle v, \lambda\rangle) c, \quad \lambda \in L,
\end{aligned}
$$

for $v \in V, r \in \mathbb{R}$. Note that $\langle w(f), w(g)\rangle=\langle f, g\rangle$ for all $f, g \in \widehat{V}$ and $w \in W_{L}$. In the case that $L=Q^{\vee}, W_{L}=W_{Q^{\vee}}=W_{0} \ltimes Q^{\vee}$ is the affine Weyl group. The extended affine Weyl group is $W_{P^{\vee}}=W_{0} \ltimes P^{\vee}$, and we will simply denote it by $W$.

Associated with the reduced irreducible finite root system $R$, there is a reduced irreducible affine root system $S=S(R):=\{\alpha+r c \mid \alpha \in R, r \in \mathbb{Z}\}$ in $\widehat{V}$. For $a \in S$, let $s_{a}: V \rightarrow V$ be the reflection in the hyperplane $a^{-1}(\{0\})$, given by

$$
s_{a}(v)=v-a(v) D a^{\vee}
$$

for $v \in V$. Then, $s_{a}=s_{D a} \mathrm{t}\left(a(0) D a^{\vee}\right) \in W_{Q^{\vee}}$. Note that $S \subset \widehat{V}$ is $W$-invariant. We define an ordered basis $\left(a_{0}, \ldots, a_{N}\right)$ of $S$ by setting

$$
\left(a_{0}, a_{1}, \ldots, a_{N}\right):=\left(-\phi+c, \alpha_{1}, \ldots, \alpha_{N}\right) .
$$

Write $S_{+}$and $S_{-}$for the associated sets of positive and negative affine roots, respectively. Note that

$$
S_{+}:=\{\alpha+r c \mid \alpha \in R, r \geq \chi(\alpha)\},
$$


where $\chi$ is the characteristic function of $R_{-}$, i.e., $\chi(\alpha)=1$ if $\alpha \in R_{-}$, and $\chi(\alpha)=0$ if $\alpha \in R_{+}$.

We put $s_{i}:=s_{a_{i}} \in W_{Q^{\vee}} \subseteq W$ for $i=0, \ldots, N$. The affine Weyl group $W_{Q^{\vee}}$ is a Coxeter group with Coxeter generators the simple reflections $s_{i}$. For $w \in W$ write $S(w):=S_{+} \cap w^{-1} S_{-}$. The length function $\ell$ on $W$ is defined by

$$
\ell(w):=\# S(w), \quad w \in W
$$

The unique element with maximal length in $W_{0}$ is denoted by $w_{0}$.

The finite abelian subgroup $\Omega:=\{w \in W \mid \ell(w)=0\}$ of $W$ is isomorphic to $P^{\vee} / Q^{\vee}$, and we have

$$
W \simeq W_{Q^{\vee} \rtimes \Omega}
$$

The action of $\Omega$ on $\widehat{V}$ restricts to a faithful action on the set $\left\{a_{0}, \ldots, a_{N}\right\}$ of simple roots of $S$, so we can view $\Omega$ as a group of permutations on the set of indices $\{0, \ldots, N\}$. We write $\mathbb{C}[\Omega]$ for the group algebra of $\Omega$.

The Bruhat order $\leq$ on $W_{Q^{\vee}}$ extends to a partial order on $W$, referred to as the Bruhat order on $W$ (cf. [14, §2.3]). It is defined as follows. For $w=\omega u$ and $w^{\prime}=\omega^{\prime} u^{\prime}$ with $\omega, \omega^{\prime} \in \Omega$ and $u, u^{\prime} \in W_{Q^{\vee}}$, we have by definition

$$
w \leq w^{\prime} \Longleftrightarrow \omega=\omega^{\prime} \text { and } u \leq u^{\prime}
$$

\subsection{Algebra of $q$-difference reflection operators}

Consider the complex torus $T:=\operatorname{Hom}_{\mathbb{Z}}\left(P^{\vee}, \mathbb{C}^{\times}\right)$. By transposition, the natural action of $W_{0}$ on $P^{\vee}$ gives rise to an action of $W_{0}$ on $T$. Fix $0<q<1$. For $\lambda \in P^{\vee}$, let $q^{\lambda} \in T$ be defined by

$$
q^{\lambda}(\mu):=q^{\langle\lambda, \mu\rangle}, \quad \mu \in P^{\vee} .
$$

The action of $W_{0}$ on $T$ extends to an action of $W=W_{0} \ltimes P^{\vee}$ on $T$ by letting $\lambda \in P^{\vee}$ act via $t \mapsto q^{\lambda} t$. Let the evaluation of $t \in T$ in a point $\lambda \in P^{\vee}$ be denoted by $t^{\lambda} \in \mathbb{C}^{\times}$. Then, summarizing, we have an action of $W$ on $T$ given by

$$
\begin{aligned}
(w t)^{\mu} & =t^{w^{-1} \mu}, \\
(\mathrm{t}(\lambda) t)^{\mu} & =q^{\langle\lambda, \mu\rangle} t^{\mu},
\end{aligned}
$$

for $t \in T, w \in W_{0}$ and $\lambda, \mu \in P^{\vee}$.

Let $\left\{\varpi_{i}^{\vee}\right\}_{i=1}^{N}$ be the set of fundamental coweights in $P^{\vee}$ with respect to $\left\{\alpha_{j}\right\}_{=1}^{N}$, so $\left\langle\varpi_{i}^{\vee}, \alpha_{j}\right\rangle=\delta_{i j}$ for $1 \leq i, j \leq N$. We identify $T \simeq(\mathbb{C} \backslash\{0\})^{N}$ via $t \leftrightarrow\left(t_{1}, \ldots, t_{N}\right)$ defined by

$$
t_{i}:=t^{\varpi_{i}^{\vee}}
$$


for $i=1, \ldots, N$. Under this identification, the action of $P^{\vee}$ on $T$ reads

$$
\mathrm{t}(\lambda) t=q^{\lambda} t=\left(q^{\left\langle\lambda, \varpi_{1}^{\vee}\right\rangle} t_{1}, \ldots, q^{\left\langle\lambda, \varpi_{N}^{\vee}\right\rangle} t_{N}\right)
$$

for $\lambda \in P^{\vee}$ and $t=\left(t_{1}, \ldots, t_{N}\right) \in T$.

The algebra of complex-valued regular functions on $T$ is $\mathbb{C}\left[x_{1}^{ \pm 1}, \ldots, x_{N}^{ \pm 1}\right]=$ $\operatorname{span}_{\mathbb{C}}\left\{x^{\lambda}\right\}_{\lambda \in P^{\vee}}$, where $x_{i}$ is the coordinate function $x_{i}(t):=t^{\varpi_{i}^{\vee}}(i=1, \ldots, N)$ and $x^{\lambda}(t):=t^{\lambda}$ for $\lambda \in P^{\vee}$. Clearly, it is isomorphic to the group algebra $\mathbb{C}\left[P^{\vee}\right]$ of $P^{\vee}$. We write $\mathbb{C}[T]=\mathbb{C}\left[x_{1}^{ \pm 1}, \ldots, x_{N}^{ \pm 1}\right]$, and we let $\mathbb{C}(T)$ denote the field of rational functions on $T, \mathcal{O}(T)$ the ring of analytic functions on $T$, and $\mathcal{M}(T)$ the field of meromorphic functions on $T$. The $W$-action on $T$ gives rise to a $W$-action by algebra automorphisms on each of these function algebras, via

$$
(w f)(t)=f\left(w^{-1} t\right)
$$

for $w \in W, t \in T$ and $f$ a (regular, rational or meromorphic) function on $T$. Note that for $\lambda \in P^{\vee}$ and $r \in \mathbb{R}$, we have

$$
w\left(x^{\lambda+r c}\right)=x^{w(\lambda+r c)}
$$

where $x^{\lambda+r c}:=q^{r} x^{\lambda} \in \mathbb{C}[T]$.

By means of this $W$-action by field automorphisms on $\mathbb{C}(T)$, we can form the smash product algebra $\mathbb{C}(T) \#_{q} W$, which we call the algebra of $q$-difference reflection operators with coefficients in $\mathbb{C}(T)$, since it acts canonically on $\mathbb{C}(T)$ and $\mathcal{M}(T)$ as $q$-difference reflection operators. For $f \in \mathbb{C}(T)$, we will write $f(X) \in \mathbb{C}(T) \#_{q} W$ for the operator on $\mathcal{M}(T)$ (or $\mathbb{C}(T)$ ) defined as multiplication by $f$. We will also write $X^{\lambda+r c}=q^{r} X^{\lambda}$ for $\lambda \in P^{\vee}$ and $r \in \mathbb{R}$.

Remark 2.1 Note that since $(\mathrm{t}(\lambda) f)(t)=f\left(q^{-\left\langle\varpi_{1}^{\vee}, \lambda\right\rangle} t_{1}, \ldots, q^{-\left\langle\varpi_{N}^{\vee}, \lambda\right\rangle} t_{N}\right)\left(\lambda \in P^{\vee}\right.$, $f \in \mathcal{M}(T)), \mathbb{C}(T) \#_{q} W$ actually depends on a choice for $q^{\frac{1}{m}}$, where $m \in \mathbb{N}$ is determined by $m\left\langle P^{\vee}, P^{\vee}\right\rangle=\mathbb{Z}$. Our global convention concerning real powers of positive real numbers justifies the apparent abuse of notation writing $q$ instead of $q^{1 / m}$.

\subsection{The extended affine Hecke algebra and Cherednik's basic representation}

Let $k_{i}(i=0, \ldots, N)$ be nonzero complex numbers such that $k_{i}=k_{j}$ if $s_{i}$ and $s_{j}$ are conjugate in $W$. Write $\underline{k}$ for the corresponding multiplicity label $\underline{k}: S \rightarrow \mathbb{C} \backslash\{0\}$, so $\underline{k}(a)=k_{i}$ for all $a \in W\left(a_{i}\right)(i=0, \ldots, N)$. We set $k_{a}:=\underline{k}(a)$ for $a \in S$. Furthermore, for $w \in W$ we define

$$
k(w):=\prod_{a \in S(w)} k_{a} .
$$


A coweight $\lambda \in P^{\vee}$ is called dominant if $\left\langle\lambda, \alpha_{i}\right\rangle \geq 0$ for $i=1, \ldots, N$. Let $P_{+}^{\vee}$ denote the set of dominant coweights.

Lemma 2.2 For $\lambda \in P_{+}^{\vee}$, we have

$$
k(\mathrm{t}(\lambda))=\prod_{\alpha \in R_{+}} k_{\alpha}^{\langle\lambda, \alpha\rangle}=\delta_{\underline{k}}^{\lambda},
$$

where $\delta_{\underline{k}} \in T$ is defined by $\left(\delta_{\underline{k}}\right)_{i}=\prod_{\alpha \in R_{+}} k_{\alpha}^{\left\langle\varpi_{i}^{\vee}, \alpha\right\rangle}(i=1, \ldots, N)$.

Proof For $\lambda \in P_{+}^{\vee}$ we have

$$
S(\mathrm{t}(\lambda))=\left\{\alpha+r c \mid \alpha \in R_{+}, 0 \leq r<\langle\lambda, \alpha\rangle\right\},
$$

cf. [14, §2.4]. Note that $k_{\alpha+r c}=k_{\alpha}$ for $\alpha \in R$ and $r \in \mathbb{Z}$ since $\alpha+r c$ and $\alpha$ are conjugate under the action of $W$. Indeed, for $\mu \in P^{\vee}$, we have $\mathrm{t}(\mu)(\alpha+r c)=$ $\alpha+(r-\langle\mu, \alpha\rangle) c$ and for any $\alpha \in R$, there exists some $v \in P^{\vee}$ such that $\langle\nu, \alpha\rangle=1$, so that we can take $\mu=r \nu$. Therefore,

$$
k(\mathrm{t}(\lambda))=\prod_{\substack{\alpha \in R_{+} \\ 0 \leq r<\langle\lambda, \alpha\rangle}} k_{\alpha+r c}=\prod_{\alpha \in R_{+}} k_{\alpha}^{\langle\lambda, \alpha\rangle}
$$

The second equality in (2.3) follows from the definitions.

Definition 2.3 The affine Hecke algebra $H_{Q \vee}$ associated with the Coxeter system $\left(W_{Q^{\vee}},\left\{s_{0}, \ldots, s_{N}\right\}\right)$ and the multiplicity label $\underline{k}$ is the unital complex associative algebra generated by elements $T_{0}, \ldots, T_{N}$, such that

(i) $T_{0}, \ldots, T_{N}$ satisfy the braid relations, i.e. if for $i \neq j$, we have

$$
s_{i} s_{j} s_{i} \cdots=s_{j} s_{i} s_{j} \cdots,
$$

with $m_{i j}$ factors on each side, then

$$
T_{i} T_{j} T_{i} \cdots=T_{j} T_{i} T_{j} \cdots,
$$

with $m_{i j}$ factors on each side;

(ii) $\left(T_{j}-k_{j}\right)\left(T_{j}+k_{j}^{-1}\right)=0$, for $j=0, \ldots, N$.

Note that since $\underline{k}$ is $W$-invariant, the group $\Omega$ acts on $H_{Q^{\vee}}$ by algebra automorphisms via $T_{i} \mapsto T_{\omega(i)}$ for $i=0, \ldots, N$.

Definition 2.4 The extended affine Hecke algebra $H=H(\underline{k})$ is the smash product $H:=H_{Q} \vee \#$. 
For $w \in W$ and a reduced expression $w=\omega s_{i_{1}} \ldots s_{i_{\ell(w)}}$ with $\omega \in \Omega$ and $i_{k} \in\{0, \ldots, N\}$, we define

$$
T_{w}:=\omega T_{i_{1}} \ldots T_{i_{\ell(w)}} \in H
$$

which is independent of the reduced expression chosen. The set $\left\{T_{w} \mid w \in W\right\}$ is a linear basis of $H$. Note that for $\underline{k} \equiv 1$, the extended affine Hecke algebra is just the group algebra $\mathbb{C}[W]$ of $W$. The finite Hecke algebra is the subalgebra $H_{0}=H_{0}(\underline{k})$ of $H$, generated by $T_{1}, \ldots, T_{N}$.

For $\lambda \in P_{+}^{\vee}$, put

$$
Y^{\lambda}:=T_{\mathrm{t}(\lambda)} \in H
$$

and for arbitrary $\lambda \in P^{\vee}$ put

$$
Y^{\lambda}:=Y^{\mu}\left(Y^{v}\right)^{-1}
$$

if $\lambda=\mu-v$ with $\mu, v \in P_{+}^{\vee}$. Then, the $Y^{\lambda}\left(\lambda \in P^{\vee}\right)$ are well defined, and we have $Y^{0}=1$ and $Y^{\lambda} Y^{\mu}=Y^{\lambda+\mu}=Y^{\mu} Y^{\lambda}$ for all $\lambda, \mu \in P^{\vee}$. Set $Y_{i}:=Y^{\varpi_{i}^{\vee}}$ for $i=1, \ldots, N$.

For $\kappa \in \mathbb{C} \backslash\{0\}$, we define the functions $b(z, \kappa)$ and $c(z, \kappa)$ by

$$
\begin{aligned}
& b(z ; \kappa):=\frac{\kappa-\kappa^{-1}}{1-z}, \\
& c(z ; \kappa):=\frac{\kappa^{-1}-\kappa z}{1-z},
\end{aligned}
$$

as rational functions in $z$. Then for $a \in S$, we define $b_{a ; \underline{k}, q}=b_{a} \in \mathbb{C}(T)$ and $c_{a ; k, q}=c_{a} \in \mathbb{C}(T)$ by

$$
\begin{aligned}
& b_{a}(t):=b\left(t^{a^{\vee}} ; k_{a}\right) \\
& c_{a}(t):=c\left(t^{a^{\vee}} ; k_{a}\right) .
\end{aligned}
$$

Remark 2.5 The $q$-dependence of $b_{a, \underline{k}, q}$ and $c_{a ; \underline{k}, q}$ comes from the convention $t^{\alpha+r c}=q^{r} t^{\alpha}$ for $\alpha \in R$ and $r \in \mathbb{R}$. If we write $\underline{k}^{-1}$ for the multiplicity label defined by $\underline{k}^{-1}(a)=(\underline{k}(a))^{-1}$ for all $a \in S$, then we have

$$
c_{a ; \underline{k}, q}\left(t^{-1}\right)=c_{a ; \underline{k}^{-1}, q^{-1}}(t)
$$

for all $a \in S$ and $t \in T$. We leave out the subscripts $\underline{k}$ and $q$ as long as there is no chance of confusion (which is until Sect. 6).

Note that $b_{a}(t)=k_{a}-c_{a}(t)$ and $\left(w c_{a}\right)(t)=c_{w(a)}(t)$ for all $w \in W$. It is convenient to introduce the notations $b_{j}:=b_{a_{j}}$ and $c_{j}:=c_{a_{j}}$ for $j=0, \ldots, N$. The following characterization of $H$ is due to Bernstein and Zelevinsky (see e.g., [14, $\S 4.2])$. 
Theorem 2.6 The affine Hecke algebra $H=H(\underline{k})$ is the unique complex associative algebra, such that

(i) $H_{0} \otimes \mathbb{C}[T] \simeq H$ as complex vector spaces, via $h \otimes f \mapsto h f(Y)$ for $h \in H_{0}, f \in$ $\mathbb{C}[T]$, where $f(Y)=\sum_{\lambda} a_{\lambda} Y^{\lambda}$ if $f=\sum_{\lambda} a_{\lambda} x^{\lambda} \in \mathbb{C}[T]$;

(ii) the canonical maps $H_{0}, \mathbb{C}[T] \hookrightarrow H$ are algebra embeddings; we write $\mathbb{C}_{Y}[T]=$ $\operatorname{span}_{\mathbb{C}}\left\{Y^{\lambda}\right\}_{\lambda \in P^{\vee}}$ for the image of $\mathbb{C}[T]$ in $H$;

(iii) Lusztig's relations are satisfied, that is,

$$
f(Y) T_{j}=T_{j}\left(s_{j} f\right)(Y)+b_{j}\left(Y^{-1}\right)\left(f(Y)-\left(s_{j} f\right)(Y)\right)
$$

for $j=1, \ldots, N$ and $f \in \mathbb{C}[T]$.

Remark 2.7 Note that $b_{j}\left(Y^{-1}\right)\left(f(Y)-\left(s_{j} f\right)(Y)\right) \in \mathbb{C}_{Y}[T]$ although $b_{j}\left(Y^{-1}\right)$ by itself is not defined as an element of $H$.

We end this section with the definition of the double affine Hecke algebra and state some of its key properties. All of this is due to Cherednik; see [5]. It starts with the realization of the affine Hecke algebra inside the algebra $\mathbb{C}(T) \#_{q} W$ of $q$-difference reflection operators (see [14, (4.3.10)]).

Theorem 2.8 There is an injective algebra homomorphism $\rho=\rho_{\underline{k}, q}: H \rightarrow$ $\mathbb{C}(T) \#_{q} W$ satisfying

$$
\begin{array}{crl}
\rho\left(T_{i}\right) & =k_{i}+c_{i}(X)\left(s_{i}-1\right), & i=0, \ldots, N, \\
\rho(\omega) & =\omega, & \omega \in \Omega .
\end{array}
$$

Remark 2.9 The image $\rho(H)$ preserves $\mathbb{C}[T]$, viewed as a subspace of the canonical $\mathbb{C}(T) \#_{q} W$-module $\mathbb{C}(T)$. The resulting faithful representation of $H$ on $\mathbb{C}[T]$ is called the basic representation of $H$.

Definition 2.10 The double affine Hecke algebra $\mathbb{H}=\mathbb{H}(\underline{k}, q)$ is the subalgebra of $\mathbb{C}(T) \#_{q} W$ generated by $H$ (i.e. by $\left.\rho_{\underline{k}, q}(H)\right)$ and by the multiplication operators $f(X)$ $(f \in \mathbb{C}[T])$.

Remark 2.11 Note that $\rho=\rho_{\underline{k}, q}$ and $\mathbb{H}=\mathbb{H}(\underline{k}, q)$ actually depend on $q^{\frac{1}{m}}$ (see Remark 2.1).

We view $\mathbb{H}$ as a left $\mathbb{C}[T]$-module by $(f, h) \mapsto f(X) h(f \in \mathbb{C}[T], h \in \mathbb{H})$. The rule $f \otimes h \mapsto f(X) h(h \in H, f \in \mathbb{C}[T])$ induces an isomorphism of $\mathbb{C}[T]$-modules

$$
\mathbb{C}[T] \otimes H \simeq \mathbb{H}
$$

Similarly to Theorem 2.6, the algebra structure of $\mathbb{H}$ can be described in terms of the left-hand side of (2.6), allowing for an abstract definition of $\mathbb{H}$ (see [4, Thm. 2.1]): 
Theorem 2.12 The double affine Hecke algebra $\mathbb{H}$ can be characterized as the unique associative algebra satisfying

(i) $\mathbb{C}[T] \otimes H \simeq \mathbb{H}$ as complex vector spaces;

(ii) the canonical maps $H, \mathbb{C}[T] \hookrightarrow \mathbb{H}$ are algebra embeddings;

(iii) the following cross relations are satisfied: for $f \in \mathbb{C}[T]$

$$
\begin{aligned}
T_{j} f(X) & =\left(s_{j} f\right)(X) T_{j}+b_{j}(X)\left(f(X)-\left(s_{j} f\right)(X)\right), \quad j=0, \ldots, N, \\
\omega f(X) & =(\omega f)(X) \omega, \quad \omega \in \Omega .
\end{aligned}
$$

A crucial ingredient in the construction of the bispectral quantum $\mathrm{KZ}$ equations is Cherednik's duality involution on $\mathbb{H}$ (see [14, (4.7.6)]).

Theorem 2.13 There exists an involutive anti-automorphism $*: \mathbb{H} \rightarrow \mathbb{H}$ determined by

$$
\begin{aligned}
T_{w}^{*} & =T_{w^{-1}}, & & w \in W_{0}, \\
\left(Y^{\lambda}\right)^{*} & =X^{-\lambda}, & & \lambda \in P^{\vee}, \\
\left(X^{\lambda}\right)^{*} & =Y^{-\lambda}, & & \lambda \in P^{\vee} .
\end{aligned}
$$

\section{Bispectral quantum KZ equations}

In this section, we extend the construction of the bispectral quantum KnizhnikZamolodchikov equations for $\mathrm{GL}_{N}$ [18] to arbitrary root systems. First, we recall Cherednik's construction of the quantum affine Knizhnik-Zamolodchikov equations [1] associated with a finite-dimensional $H$-module.

\subsection{The quantum affine KZ equations}

In order to define the quantum affine $\mathrm{KZ}$ equations, we first need to left-localize the double affine Hecke algebra $\mathbb{H} \simeq \mathbb{C}[T] \otimes H$ (see Theorem 2.12) with respect to $\mathbb{C}[T] \backslash\{0\}$. As a complex vector space, the resulting algebra $\widehat{\mathbb{H}}$ can be defined as $\widehat{\mathbb{H}} \simeq \mathbb{C}(T) \otimes H$, and then its algebra structure is determined by requiring $\mathbb{C}(T)$ and $H$ to be subalgebras and by requiring the cross relations (2.7) and (2.8) to hold for $f \in \mathbb{C}(T)$.

The injective map $\rho$ of Theorem 2.8 extends to an injective algebra homomorphism

$$
\rho: \widehat{\mathbb{H}} \rightarrow \mathbb{C}(T) \#_{q} W
$$

by setting $\rho(f(X))=f(X)$ for $f \in \mathbb{C}(T)$. Note that $\rho\left(c_{j}(X)^{-1}\left(T_{j}-b_{j}(X)\right)\right)=s_{j}$ for $0 \leq j \leq N$, which shows that $\rho$ is surjective and therefore establishes an isomorphism $\widehat{\mathbb{H}} \simeq \mathbb{C}(T) \#_{q} W$. Restricting the inverse $\rho^{-1}$ to $W$ gives a realization of $W$ inside $\widehat{\mathbb{H}^{\times}}$. 
The left multiplication map turns $H$ into a left module over itself. The action of $\widehat{\mathbb{H}}$ on the induced module Ind $\widehat{\mathbb{H}}(H)=\widehat{\mathbb{H}} \otimes_{H} H$ can be pushed forward along the linear isomorphism $\widehat{\mathbb{H}} \otimes_{H} H \simeq \mathbb{C}(T) \otimes H$ to obtain an algebra homomorphism

$$
\pi=\pi_{\underline{k}}: \widehat{\mathbb{H}} \rightarrow \operatorname{End}(\mathbb{C}(T) \otimes H) .
$$

We regard $\mathbb{C}(T) \#_{q} W \otimes H$ as a subalgebra of $\operatorname{End}(\mathbb{C}(T) \otimes H)$ by letting $\mathbb{C}(T) \#_{q} W$ act on $\mathbb{C}(T)$ as in Sect. 2.2 and $H$ on $H$ by left multiplication. Then, the pullback $\tau_{x}=\tau_{x, k}:=\pi \circ \rho^{-1}$ of $\pi$ along $\rho^{-1}$ is an algebra homomorphism

$$
\tau_{x}: \mathbb{C}(T) \#_{q} W \rightarrow \mathbb{C}(T) \#_{q} W \otimes H \subset \operatorname{End}(\mathbb{C}(T) \otimes H),
$$

which is explicitly given by

$$
\begin{aligned}
& \tau_{x}(f)=f(X) \otimes 1, \quad f \in \mathbb{C}(T), \\
& \tau_{x}\left(s_{j}\right)=\left(c_{j}(X)^{-1} \otimes 1\right)\left(s_{j} \otimes T_{j}-b_{j}(X) s_{j} \otimes 1\right), \quad 0 \leq j \leq N, \\
& \tau_{x}(\omega)=\omega \otimes \omega, \quad \omega \in \Omega \text {, }
\end{aligned}
$$

as can be verified by a direct computation using the formula for $\rho^{-1}$ and the cross relations (2.7).

Remark 3.1 The reason for the subscript $x$ in $\tau_{x}$ will become apparent in the next subsection when we discuss the bispectral story. Then, two copies of $T$ will play a role and $x$ will denote the coordinate functions on one of them.

Note that $\tau_{x}\left(s_{j}\right)=F_{s_{j}}(X)\left(s_{j} \otimes 1\right)$ with $F_{s_{j}}(X)=\left(c_{j}(X)^{-1} \otimes 1\right)\left(1 \otimes T_{j}-b_{j}(X) \otimes\right.$ $1) \in \mathbb{C}(T) \otimes H$ and trivially also $\tau_{x}(\omega)=F_{\omega}(X)(\omega \otimes 1)$ with $F_{\omega}=1 \otimes \omega \in \mathbb{C}(T) \otimes H$. In fact, more generally, we have

$$
\tau_{x}(w)=F_{w}(X)(w \otimes 1), \quad w \in W
$$

where $F_{w}$ are $H$-valued rational functions on $T$ satisfying

$$
F_{e}(t)=1, \quad F_{v w}(t)=F_{v}(t) F_{w}\left(v^{-1} t\right)
$$

for all $v, w \in W$ and $t \in T$. Viewed as elements of $\operatorname{End}(\mathbb{C}(T) \otimes H)$ the $F_{w}(X)$ $(w \in W)$ are $\mathbb{C}(T)$-linear and invertible (indeed $\left.F_{w}^{-1}(X)=\left(w^{-1} \otimes 1\right) \tau_{x}\left(w^{-1}\right)\right)$. In the language of non-abelian group cohomology, (3.1) means that $w \mapsto F_{w}(X)$ constitutes a cocycle $W \rightarrow \mathrm{GL}_{\mathbb{C}(T)}(\mathbb{C}(T) \otimes H)$, where $\mathrm{GL}_{\mathbb{C}(T)}(\mathbb{C}(T) \otimes H)$ is a $W$-group via the usual action of $W$ on the first tensor leg of $\mathbb{C}(T) \otimes \operatorname{End}(H) \simeq \mathrm{GL}_{\mathbb{C}(T)}(\mathbb{C}(T) \otimes H)$.

Now let $M$ be a left module over the affine Hecke algebra $H$. Then, $M^{\mathcal{M}(T)}=$ $\mathcal{M}(T) \otimes M$ is a module over $\mathbb{C}(T) \#_{q} W \otimes H$, where $\mathbb{C}(T) \#_{q} W$ acts on $\mathcal{M}(T)$ as described in Sect. 2.2. Consequently, $\tau_{x}$ gives rise to a representation

$$
\tau_{x}^{M}: W \rightarrow \mathrm{GL}\left(M^{\mathcal{M}(T)}\right)
$$


defining $\tau_{x}^{M}(w)(w \in W)$ to be $\tau_{x}(w) \in \mathbb{C}(T) \#_{q} W \otimes H$ acting on $M^{\mathcal{M}(T)}$. Let $F_{w}^{M}$ $(w \in W)$ denote the corresponding functions $F_{w} \in \mathbb{C}(T) \otimes H$ acting on $M^{\mathcal{M}(T)}$. For simplicity, we write $F_{\lambda}^{M}=F_{\mathrm{t}(\lambda)}^{M}$ for $\lambda \in P^{\vee}$.

Definition 3.2 (Cherednik [1]) The $q$-difference equations

$$
F_{\lambda}^{M}(t) f\left(q^{-\lambda} t\right)=f(t), \quad \lambda \in P^{\vee}
$$

for $f \in \mathcal{M}(T) \otimes M$, are called the quantum affine $\mathrm{KZ}$ (qKZ) equations for the $H$-module $M$.

From the cocycle condition (3.1) and the fact that $P^{\vee}$ is an abelian subgroup of $W$, it follows immediately that the qKZ equations form a holonomic system of $q$-difference equations, that is,

$$
F_{\lambda}^{M}(t) F_{\mu}^{M}\left(q^{-\lambda} t\right)=F_{\mu}^{M}(t) F_{\lambda}^{M}\left(q^{-\mu} t\right)
$$

for all $\lambda, \mu \in P^{\vee}$.

In this paper, we will restrict our attention to a particular representation of $H$. Recall that $H \simeq H_{0} \otimes \mathbb{C}_{Y}[T]$ (cf. Theorem 2.6). Fix $\zeta \in T$ and let $\chi_{\zeta}: \mathbb{C}_{Y}[T] \rightarrow \mathbb{C}$ be the evaluation character $f(Y) \mapsto f(\zeta)$ for $f \in \mathbb{C}[T]$. We define $M_{\zeta}$ to be the induced $H$-module $M_{\zeta}:=\operatorname{Ind}_{\mathbb{C}_{Y}[T]}^{H}\left(\chi_{\zeta}\right)=H \otimes_{\chi_{\zeta}} \mathbb{C}$. It is the minimal principal series representation of $H$ with central character $\zeta$. As complex vector spaces we identify $M_{\zeta} \simeq H_{0}$ via

$$
T_{w} \otimes_{\chi_{\zeta}} 1 \mapsto T_{w}, \quad\left(w \in W_{0}, f \in \mathbb{C}[T]\right)
$$

The qKZ equations corresponding to $M_{\zeta}$ thus can be viewed as a holonomic system of $q$-difference equations for meromorphic functions $f(t)$ on $T$ with values in $H_{0}$. Now $\mathbb{H} \simeq \mathbb{C}[T] \otimes H$, so that since $H \simeq H_{0} \otimes \mathbb{C}[T]$, the double affine Hecke algebra $\mathbb{H}$ contains another copy of $\mathbb{C}[T]$. In view of Cherednik's duality anti-automorphism, one might ask, when $\zeta$ is considered as a variable $\gamma$ on the second torus, whether one can find a set of $q$-difference equations acting on this central character $\gamma$, such that together with the original $\mathrm{qKZ}$ equations it makes up a holonomic system of $q$-difference equations for meromorphic functions $f(t, \gamma)$ on $T \times T$ with values in $H_{0}$. The answer turns out to be positive. The idea is as follows.

The construction of the $\mathrm{qKZ}$ equations depended on the realization of $W$ inside the localization of $\mathbb{H}$ by sending the $w$ to the so-called normalized intertwiners $\rho^{-1}(w)$. Of course, we can multiply these intertwiners by appropriate factors from $\mathbb{C}[T]$ to obtain elements $\widetilde{S}_{w}$ which do live in $\mathbb{H}$. Clearly, the map $W \rightarrow \mathbb{H}^{\times}, w \mapsto \widetilde{S}_{w}$ will no longer be a group homomorphism (like $\rho^{-1}$ ), but the $\widetilde{S}_{w}$ still serve as intertwining elements from which a cocycle can be constructed. Then, Cherednik's duality antiautomorphism can be invoked to obtain $Y$-intertwining elements and extend the cocycle to a 'double cocycle' which will give rise to the bispectral quantum KZ equations. This is explained in the following subsection. 


\subsection{Bispectral quantum KZ equations}

The construction of the bispectral quantum $\mathrm{KZ}$ equations in the present setting is more or less the same as in the $\mathrm{GL}_{N}$ case, which was done in $[18, \S 3]$. Here, we repeat the construction, but, since it is a matter of simply adapting the notations from [18], we omit the proofs.

In view of the last paragraph of the previous subsection, we should first renormalize the intertwiners so that they become members of $\mathbb{H}$. We put

$$
\begin{aligned}
\widetilde{S}_{i} & :=\left(k_{i}-k_{i}^{-1} X^{-a_{i}^{\vee}}\right) s_{i} \in \mathbb{C}(T) \#_{q} W, \quad i=0, \ldots, N \\
\widetilde{S}_{\omega} & :=\omega \in \mathbb{C}(T) \#_{q} W, \quad \omega \in \Omega,
\end{aligned}
$$

giving rise to the renormalized intertwiners $\widetilde{S}_{w}(w \in W)$, defined in the following proposition (see also [5, §1.3]).

Proposition 3.3 Let $w=s_{i_{1}} \ldots s_{i_{r}} \omega$ be a reduced expression for $w \in W$, with $i_{1}, \ldots, i_{r} \in\{0, \ldots, N\}, \omega \in \Omega$. Then,

(i) $\widetilde{S}_{w}:=\widetilde{S}_{i_{1}} \ldots \widetilde{S}_{i_{r}} \widetilde{S}_{\omega}$ is a well-defined element of $\mathbb{C}(T) \# W$;

(ii) $\widetilde{S}_{w} \in \mathbb{H}$, in particular $\widetilde{S}_{i}=\left(1-X^{-a_{i}^{\vee}}\right) T_{i}+\left(k_{i}-k_{i}^{-1}\right) X^{-a_{i}^{\vee}}(0 \leq i \leq N)$;

(iii) the $\widetilde{S}_{i}(i=0, \ldots, N)$ satisfy the braid relations $(c f$. Definition 2.3(i));

(iv) $\widetilde{S}_{w} f(X)=(w f)(X) \widetilde{S}_{w}$ for $w \in W, f \in \mathbb{C}[T]$;

(v) $\widetilde{S}_{i} \widetilde{S}_{i}=\left(k_{i}-k_{i}^{-1} X^{a_{i}^{\vee}}\right)\left(k_{i}-k_{i}^{-1} X^{-a_{i}^{\vee}}\right)$ for $i=0, \ldots N$.

For $0 \leq i \leq N$ define $d_{i} \in \mathbb{C}[T]$ by $d_{i}(t):=\left(k_{i}-k_{i}^{-1} t^{-a_{i}^{\vee}}\right)$. Then for $w \in W$ as in the proposition, we have

$$
\widetilde{S}_{w}=d_{i_{1}}(X)\left(s_{i_{1}} d_{i_{2}}\right)(X) \ldots\left(s_{i_{1}} \ldots s_{i_{r-1}} d_{i_{r}}\right)(X) w
$$

The proof of part (i) of the proposition relies on the fact that

$$
d_{w}:=d_{i_{1}}\left(s_{i_{1}} d_{i_{2}}\right) \ldots\left(s_{i_{1}} \ldots s_{i_{r-1}} d_{i_{r}}\right)
$$

is independent of the reduced expression for $w$ (see e.g., [14, (2.2.9)]).

Now the 'double cocycle' we are going to construct is a cocycle of $W \times W$. In fact, it turns out to be convenient to anticipate the role that the anti-involution of $\mathbb{H}$ will play and extend $W \times W$ as follows. Note that the two-group $\mathbb{Z}_{2}$ acts on $W \times W$ by $\iota\left(w, w^{\prime}\right)=\left(w^{\prime}, w\right)$, where $\iota \in \mathbb{Z}_{2}$ denotes the nontrivial element. Then, we put

$$
\mathbb{W}:=\mathbb{Z}_{2} \ltimes(W \times W) .
$$

Furthermore, the cocycle will act on $H_{0}$-valued meromorphic functions on $T \times T$. Let us write $\mathbb{K}:=\mathcal{M}(T \times T)$ for the field of meromorphic functions on $T \times T$. Moreover, write $\mathbb{L}:=\mathbb{C}[T] \otimes \mathbb{C}[T] \simeq \mathbb{C}[T \times T]$ for the ring of complex-valued regular functions on $T \times T$. It acts on $\mathbb{H}$ via

$$
(f \otimes g) \cdot h:=f(X) h g(Y)
$$


for $f, g \in \mathbb{C}[T]$ and $h \in \mathbb{H}$. We will usually write $(t, \gamma)$ for a typical point of $T \times T$. Let $x=\left(x_{1}, \ldots, x_{N}\right)$ denote the coordinate functions of the first copy of $T$ in $T \times T$ and $y=\left(y_{1}, \ldots, y_{N}\right)$ the coordinate functions of the second copy. For $f \in \mathbb{C}[T]$, we define $f(x) \in \mathbb{L}$ by the rule $(t, \gamma) \mapsto f(t)$, and $f(y) \in \mathbb{L}$ by $(t, \gamma) \mapsto f(\gamma)$. We use the same conventions for $f(x), f(y) \in \mathbb{K}$ when $f \in \mathcal{M}(T)$.

An intermediate step in the construction of a $\mathbb{W}$-action on $H^{\mathbb{K}}=\mathbb{K} \otimes H_{0}$ are the complex linear endomorphisms $\sigma_{\left(w, w^{\prime}\right)}\left(w, w^{\prime} \in W\right)$ of $\mathbb{H}$ defined by

$$
\begin{aligned}
\sigma_{\left(w, w^{\prime}\right)}(h) & =\widetilde{S}_{w} h \widetilde{S}_{w^{\prime}}^{*}, \\
\sigma_{\iota}(h) & =h^{*}
\end{aligned}
$$

for $h \in \mathbb{H}$. As a corollary of Proposition 3.3, we have

Lemma 3.4 The complex linear endomorphisms $\sigma_{\left(w, w^{\prime}\right)}$ and $\sigma_{\iota}$ of $\mathbb{H}$ satisfy:

(i) the $\sigma_{\left(s_{i}, e\right)}(i=0, \ldots, N)$ satisfy the braid relations;

(ii) $\sigma_{\left(s_{i}, e\right)}^{2}=d_{s_{i}}(x)\left(s_{i} d_{s_{i}}\right)(x) \cdot \mathrm{id} \mathbb{H}$ for $i=0, \ldots, N$;

(iii) $\sigma_{(\omega, e)} \sigma_{\left(s_{i}, e\right)} \sigma_{\left(\omega^{-1}, e\right)}=\sigma_{\left(s_{\omega(i)}, e\right)}$ for $i=0, \ldots, N$ and $\omega \in \Omega$;

(iv) $\sigma_{\iota}^{2}=\mathrm{id}_{\mathbb{H}}$ and $\sigma_{(e, w)}=\sigma_{\iota} \sigma_{(w, e)} \sigma_{\iota}$ for $w \in W$;

(v) $\sigma_{(w, e)} \sigma_{\left(e, w^{\prime}\right)}=\sigma_{\left(w, w^{\prime}\right)}=\sigma_{\left(e, w^{\prime}\right)} \sigma_{(w, e)}$ for $w, w^{\prime} \in W$.

Let us investigate the behavior of these maps under the action of $\mathbb{L}$. First consider the group involution $\diamond: W \rightarrow W$ given by $w^{\diamond}=w$ for $w \in W_{0}$ and $\lambda^{\diamond}=-\lambda$ for $\lambda \in P^{\vee}$. Then, $\mathbb{W}$ acts on $T \times T$ by

$$
\begin{aligned}
\left(w, w^{\prime}\right)(t, \gamma) & =\left(w t, w^{\prime \diamond} \gamma\right), \\
\iota(t, \gamma) & =\left(\gamma^{-1}, t^{-1}\right)
\end{aligned}
$$

for $w, w^{\prime} \in W$, where $t^{-1}:=\left(t_{1}^{-1}, \ldots, t_{N}^{-1}\right) \in T$. Transposition yields an action of $\mathbb{W}$ on $\mathbb{K}$ by field automorphisms and is given by

$$
(\mathrm{w} f)(t, \gamma)=f\left(\mathrm{w}^{-1}(t, \gamma)\right), \quad \mathrm{w} \in \mathbb{W} \text {. }
$$

Note that $\mathbb{L}=\mathbb{C}[T \times T]$ is a $\mathbb{W}$-subalgebra of $\mathbb{K}$. As a consequence of the intertwining properties of the $\widetilde{S}_{w}$, we have

Lemma 3.5 For $h \in \mathbb{H}$ and $f \in \mathbb{L}$ we have

$$
\begin{aligned}
\sigma_{\left(w, w^{\prime}\right)}(f \cdot h) & =\left(\left(w, w^{\prime}\right) f\right) \cdot \sigma_{\left(w, w^{\prime}\right)}(h), \\
\sigma_{\iota}(f \cdot h) & =(\iota f) \cdot \sigma_{\iota}(h)
\end{aligned}
$$

for $w, w^{\prime} \in W$.

As $\mathbb{L}$-modules we have $H_{0}^{\mathbb{K}} \simeq \mathbb{K} \otimes_{\mathbb{L}} \mathbb{H}$, so the lemma enables us to extend the maps $\sigma_{\left(w, w^{\prime}\right)}\left(w, w^{\prime} \in W\right)$ and $\sigma_{\iota}$ to complex linear endomorphisms of $H_{0}^{\mathbb{K}}$ for which (3.6) holds for all $f \in \mathbb{K}$ and $h \in H_{0}^{\mathbb{K}}$. Note that the properties of $\sigma_{\left(w, w^{\prime}\right)}$ and $\sigma_{\iota}$ as described in Lemma 3.4 also hold true as identities between endomorphisms of $H_{0}^{\mathbb{K}}$. 
We come to the main result of this subsection. It follows from the previous observations in the same way as the corresponding result for $\mathrm{GL}_{N}$ (see [18, Thm. 3.3]).

Theorem 3.6 There is a group homomorphism

$$
\tau: \mathbb{W} \rightarrow \mathrm{GL}_{\mathbb{C}}\left(H_{0}^{\mathbb{K}}\right)
$$

satisfying

$$
\begin{aligned}
\tau\left(w, w^{\prime}\right)(f) & =d_{w}(x)^{-1} d_{w^{\prime}}^{\diamond}(y)^{-1} \cdot \sigma_{\left(w, w^{\prime}\right)}(f), \\
\tau(\iota)(f) & =\sigma_{\iota}(f)
\end{aligned}
$$

for $w, w^{\prime} \in W$ and $f \in H_{0}^{\mathbb{K}}$. It satisfies $\tau(\mathrm{w})(g \cdot f)=\mathrm{w} g \cdot \tau(\mathrm{w})(f)$ for $g \in \mathbb{K}$, $f \in H_{0}^{\mathbb{K}}$ and $\mathrm{w} \in \mathbb{W}$.

Remark 3.7 Fix $\zeta \in T$. Let $w \in W$ and recall that we write $\tau_{x}^{M_{\zeta}}(w)$ for $\tau_{x}(w) \in$ $\mathbb{C}(T) \#_{q} W$ viewed as endomorphism of $\mathcal{M}(T) \otimes M_{\zeta}$ as explained in Sect. 3.1. Then for $w \in W, f \in \mathcal{M}(T)$ and $h \in H_{0} \simeq M_{\zeta}$ (see (3.3)), we have

$$
\tau_{x}^{M_{\zeta}}(w)(f \otimes h)=\tau(w, e)(f(x) \otimes h)(\cdot, \zeta)
$$

as $H_{0}$-valued meromorphic functions on $T$.

We are in position to define the $\mathbb{W}$-cocycle with values in $\mathrm{GL}_{\mathbb{K}}\left(H_{0}^{\mathbb{K}}\right)$, which is a $\mathbb{W}$-group by the action of $\mathbb{W}$ on the first tensor leg of $\mathbb{K} \otimes \operatorname{GL}\left(H_{0}\right) \simeq \mathrm{GL}_{\mathbb{K}}\left(H_{0}^{\mathbb{K}}\right)$ (cf. Sect. 3.1). This $\mathbb{W}$-action on $\mathrm{GL}_{\mathbb{K}}\left(H_{0}^{\mathbb{K}}\right)$ is denoted without mentioning the representation map (just as we do for the $\mathbb{W}$-action on $\mathbb{K}$, cf. (3.5)).

Corollary 3.8 The map $\mathrm{w} \mapsto C_{\mathrm{W}}:=\tau(\mathrm{w}) \mathrm{w}^{-1}$ is a cocycle of $\mathbb{W}$ with values in the $\mathbb{W}$-group $\mathrm{GL}_{\mathbb{K}}\left(H_{0}^{\mathbb{K}}\right)$. In other words, $C_{\mathrm{W}} \in \mathrm{GL}_{\mathbb{K}}\left(H_{0}^{\mathbb{K}}\right)$ and

$$
C_{\mathrm{ww}^{\prime}}=C_{\mathrm{w}}{ }_{\mathrm{w}} C_{\mathrm{w}^{\prime} \mathrm{W}^{-1}}
$$

for all $\mathrm{w}, \mathrm{w}^{\prime} \in \mathbb{W}$.

In the same way as the cocycle $F_{w}(w \in W)$ in Sect. 3.1 gave rise to the quantum KZ equations, the cocycle $C_{\mathrm{W}}(\mathrm{w} \in \mathbb{W})$ gives rise to a holonomic system of $q$-difference equations for meromorphic functions on $T \times T$ with values in $H_{0}$. By construction we have

$$
(\tau(\mathrm{w}) f)(t, \gamma)=C_{\mathrm{w}}(t, \gamma) f\left(\mathrm{w}^{-1}(t, \gamma)\right)
$$

for $\mathrm{w} \in \mathbb{W}$ and $f \in H_{0}^{\mathbb{K}}$. For the sake of simplicity, write $C_{(\lambda, \mu)}:=C_{(\mathrm{t}(\lambda), \mathrm{t}(\mu))}$ for $\lambda, \mu \in P^{\vee}$. 
Definition 3.9 We call the $q$-difference equations

$$
C_{(\lambda, \mu)}(t, \gamma) f\left(q^{-\lambda} t, q^{\mu} \gamma\right)=f(t, \gamma) \quad \forall \lambda, \mu \in P^{\vee}
$$

the bispectral quantum $\mathrm{KZ}(\mathrm{BqKZ})$ equations. We write $\mathrm{SOL}$ for the set of solutions $f \in H_{0}^{\mathbb{K}}$ of (3.9).

Let $\mathbb{F} \subset \mathbb{K}$ denote the subfield consisting of $f \in \mathbb{K}$ satisfying $(\mathrm{t}(\lambda), \mathrm{t}(\mu)) f=f$ for all $\lambda, \mu \in P^{\vee}$. Furthermore, let $\mathbb{W}_{0}$ denote the subgroup $\mathbb{Z}_{2} \ltimes\left(W_{0} \times W_{0}\right)$ of $\mathbb{W}$.

Corollary 3.10 (i) The BqKZ equations (3.9) form a holonomic system of $q$-difference equations, that is

$$
C_{(\lambda, \mu)}(t, \gamma) C_{(\nu, \xi)}\left(q^{-\lambda} t, q^{\mu} \gamma\right)=C_{(\nu, \xi)}(t, \gamma) C_{(\lambda, \mu)}\left(q^{-v} t, q^{\xi} \gamma\right)
$$

for $\lambda, \mu, \nu, \xi \in P^{\vee}$, as $\operatorname{End}\left(H_{0}\right)$-valued meromorphic functions in $(t, \gamma) \in$ $T \times T$.

(ii) The solution space SOL of BqKZ is a $\tau\left(\mathbb{W}_{0}\right)$-invariant $\mathbb{F}$-subspace of $H_{0}^{\mathbb{K}}$.

Now fix $\zeta \in T$. By construction, BqKZ (in some sense) contains Cherednik's qKZ equation associated with the principal series module $M_{\zeta}$. Concretely, in view of Remark 3.7, Cherednik's quantum KZ equation (3.2) for $M=M_{\zeta}$ is just

$$
C_{(\lambda, e)}(t, \zeta) f\left(q^{-\lambda} t\right)=f(t), \quad \forall \lambda \in P^{\vee}
$$

for $H_{0}$-valued meromorphic functions $f$ on $T$. In analogy with BqKZ, we write $\mathrm{SOL}_{\zeta} \subset H_{0}^{\mathcal{M}(T)}$ for the set of solutions of (3.11). Regarding $H_{0}^{\mathcal{M}(T)}$ as a vector space over $\mathcal{E}(T):=\left\{f \in \mathcal{M}(T) \mid \mathrm{t}(\lambda) f=f, \forall \lambda \in P^{\vee}\right\}, \operatorname{SOL}_{\zeta}$ is a $\tau_{x}^{M_{\zeta}}\left(W_{0}\right)$ invariant subspace of $H_{0}^{\mathcal{M}(T)}$.

\section{Formal principal series representation and the cocycle values}

In this section, we investigate the principal series representation $M_{\zeta}$ of $H$, when the (fixed) central character $\zeta \in T$ is regarded as a meromorphic variable. This allows us to give explicit expressions for the cocycle values of the simple reflections.

\subsection{Formal principal series representation}

Recall that $M_{\zeta}=\operatorname{Ind}_{\mathbb{C}_{Y}[T]}^{H}\left(\chi_{\zeta}\right)$. Now, we view $\mathbb{C}_{Y}[T]$ as a left $\mathbb{C}_{Y}[T]$-module by left multiplication, and we put $M:=\operatorname{Ind}_{\mathbb{C}_{Y}[T]}^{H}\left(\mathbb{C}_{Y}[T]\right)$. Let another copy of $\mathbb{C}[T] \simeq$ $\mathbb{C}[\{1\} \times T] \subset \mathbb{L}$ act on $M$ via

$$
f \cdot\left(h \otimes_{\mathbb{C}_{Y}[T]} g(Y)\right)=h \otimes_{\mathbb{C}_{Y}[T]}(f g)(Y) \quad f, g \in \mathbb{C}[T], h \in H .
$$


Note that $M \simeq \mathbb{C}[\{1\} \times T] \otimes H_{0}=H_{0}^{\mathbb{C}[\{1\} \times T]}$ as modules over $\mathbb{C}[\{1\} \times T]$, hence the representation map can be regarded as an algebra homomorphism

$$
\eta: H \rightarrow \operatorname{End}_{\mathbb{C}[\{1\} \times T]}\left(H_{0}^{\mathbb{C}[\{1\} \times T]}\right)
$$

Also note that $\operatorname{End}_{\mathbb{C}[\{1\} \times T]}\left(H_{0}^{\mathbb{C}[\{1\} \times T]}\right) \simeq \mathbb{C}[\{1\} \times T] \otimes \operatorname{End}\left(H_{0}\right)$, so we can and sometimes will regard $\eta(h)(h \in H)$ as an $\operatorname{End}\left(H_{0}\right)$-valued regular function on $T$ denoted by $\gamma \mapsto \eta(h)(\gamma)$. By extending the ground ring $\mathbb{C}[\{1\} \times T]$ to $\mathbb{K}$, we can extend $\eta$ to an algebra homomorphism

$$
\eta: H \rightarrow \operatorname{End}_{\mathbb{K}}\left(H_{0}^{\mathbb{K}}\right)
$$

Similarly, $\eta(h)$ can be viewed as an $\operatorname{End}\left(H_{0}\right)$-valued function in $(t, \gamma) \in T \times T$. As such it is constant in $t$, and in case $h \in H_{0}$ it is also constant in $\gamma$.

Before being more specific about $\eta$, we need the following concept (cf. [14, §2.6]). A subset $X$ of $P^{\vee}$ is said to be saturated if for each $\lambda \in X$ and $\alpha \in R$ we have $\lambda-r \alpha^{\vee} \in X$ for all $0 \leq r \leq\langle\lambda, \alpha\rangle$. For $\lambda \in P^{\vee}$ let $\Sigma(\lambda)$ denote the smallest saturated subset of $P^{\vee}$ that contains $\lambda$.

Lemma 4.1 For $w \in W_{0}$ and $1 \leq i \leq N$ we have

$$
\eta\left(T_{i}\right) T_{w}= \begin{cases}T_{s_{i} w} & \text { if } \ell\left(s_{i} w\right)=\ell(w)+1 \\ \left(k_{i}-k_{i}^{-1}\right) T_{w}+T_{s_{i} w} & \text { if } \ell\left(s_{i} w\right)=\ell(w)-1\end{cases}
$$

and for $p \in \mathbb{C}[T]$ we have

$$
\eta(p(Y))(\gamma) T_{e}=p(\gamma) T_{e}
$$

as regular $H_{0}$-valued functions in $\gamma$. Moreover, for $\lambda \in P^{\vee}$ and $w \in W_{0}$, we have

$$
\eta\left(Y^{\lambda}\right)(\gamma) T_{w}=\sum_{u \leq w} p_{u, w}^{\lambda}(\gamma) T_{u}
$$

where $p_{u, w}^{\lambda}(\gamma) \in \operatorname{span}_{\mathbb{C}}\left\{\gamma^{\mu}\right\}_{\mu \in \Sigma\left(\lambda_{+}\right)}$and $p_{w, w}^{\lambda}(\gamma)=\gamma^{w^{-1}(\lambda)}$.

Proof Only (4.3) requires proof. We use induction with respect to the length $\ell(w)$ of $w$, the case $\ell(w)=0$ being (4.2). Next, consider $T_{s_{i}} w$ with $\ell\left(s_{i} w\right)=\ell(w)+1$. Using (2.5), we find

$$
\begin{aligned}
\eta\left(Y^{\lambda}\right)(\gamma) T_{s_{i} w} & =\eta\left(Y^{\lambda} T_{i}\right)(\gamma) T_{w} \\
& =\eta\left(T_{i} Y^{s_{i}(\lambda)}\right)(\gamma) T_{w}+\left(k_{i}-k_{i}^{-1}\right) \eta\left(\frac{Y^{\lambda}-Y^{s_{i}(\lambda)}}{1-Y^{-\alpha_{i}^{\vee}}}\right)(\gamma) T_{w} .
\end{aligned}
$$


Considering the first term we use the induction hypothesis to find

$$
\eta\left(T_{i} Y^{s_{i}(\lambda)}\right)(\gamma) T_{w}=T_{i} \sum_{u \leq w} \tilde{p}_{u, w}^{s_{i}(\lambda)}(\gamma) T_{u}=\sum_{u \leq w} \tilde{p}_{u, w}^{s_{i}(\lambda)}(\gamma) T_{i} T_{u},
$$

with $\tilde{p}_{u, w}^{s_{i}(\lambda)}(\gamma) \in \operatorname{span}_{\mathbb{C}}\left\{\gamma^{\mu}\right\}_{\mu \in \Sigma\left(s_{i}(\lambda)_{+}\right)}$and $\tilde{p}_{w, w}^{s_{i}(\lambda)}(\gamma)=\gamma^{w^{-1}\left(s_{i}(\lambda)\right)}$. Since $\Sigma\left(s_{i}(\lambda)_{+}\right)=$ $\Sigma\left(\lambda_{+}\right)$and $w^{-1}\left(s_{i}(\lambda)\right)=\left(s_{i} w\right)^{-1}(\lambda)$, we can rewrite this as

$$
\eta\left(T_{i} Y^{s_{i}(\lambda)}\right)(\gamma) T_{w}=\sum_{u \leq s_{i} w} p_{u, s_{i} w}^{\lambda}(\gamma) T_{u}
$$

with $p_{u, s_{i} w}^{\lambda}(\gamma) \in \operatorname{span}_{\mathbb{C}}\left\{\gamma^{\mu}\right\}_{\mu \in \Sigma\left(\lambda_{+}\right)}$and $p_{s_{i} w, s_{i} w}^{\lambda}(\gamma)=\gamma^{\left(s_{i} w\right)^{-1}(\lambda)}$.

We deal with the second term, the expansion of which will consist of terms only involving $T_{u}$ with $u<s_{i} w$. Set $n:=\left\langle\lambda, \alpha_{i}\right\rangle$. Note that

$$
\frac{Y^{\lambda}-Y^{s_{i}(\lambda)}}{1-Y^{-\alpha_{i}^{\vee}}}= \begin{cases}Y^{\lambda}+Y^{\lambda-\alpha_{i}^{\vee}}+\cdots+Y^{\lambda-(n-1) \alpha_{i}^{\vee},} & n>0, \\ 0, & n=0, \\ -Y^{\lambda-n \alpha_{i}^{\vee}}-Y^{\lambda-(n+1) \alpha_{i}^{\vee}}-\cdots-Y^{\lambda+\alpha_{i}^{\vee}}, & n<0,\end{cases}
$$

which is in $\operatorname{span}_{\mathbb{C}}\left\{Y^{\mu}\right\}_{\mu \in \Sigma\left(\lambda_{+}\right)}$in all three cases. We can apply the induction hypothesis to each of the $Y^{\mu}\left(\mu \in \Sigma\left(\lambda_{+}\right)\right)$to obtain

$$
\eta\left(Y^{\mu}\right)(\gamma) T_{w}=\sum_{u \leq w} \check{p}_{u, w}^{\mu}(\gamma) T_{u}
$$

with coefficients $\check{p}_{u, w}^{\mu}(\gamma) \in \operatorname{span}_{\mathbb{C}}\left\{\gamma^{\nu}\right\}_{\nu \in \Sigma\left(\mu_{+}\right)}$. Since for each $\mu \in \Sigma\left(\lambda_{+}\right)$, we have $\mu_{+} \in \Sigma\left(\lambda_{+}\right)$, and then by [14, (2.6.3)] $\Sigma\left(\mu_{+}\right) \subset \Sigma\left(\lambda_{+}\right)$, we obtain the desired expansion.

We end this subsection by introducing a $\mathbb{K}$-basis of $H_{0}^{\mathbb{K}}$, consisting of common eigenfunctions of $\eta\left(\mathbb{C}_{Y}[T]\right)$. Note that $\widetilde{S}_{w}^{*} \in H$ for $w \in W_{0}$. Define

$$
\xi_{w}:=\eta\left(\widetilde{S}_{w^{-1}}^{*}\right) T_{e}, \quad w \in W_{0}
$$

Just as we view $\eta(h)$ as End $\left(H_{0}\right)$-valued function in different ways, we will regard $\xi_{w}$ both as regular $H_{0}$-valued function in $\gamma \in T$ and as meromorphic $H_{0}$-valued function in $(t, \gamma) \in T \times T$ (constant in $t$ ).

Lemma 4.2 $\left\{\xi_{w}\right\}_{w \in W_{0}}$ is a $\mathbb{K}$-basis of $H_{0}^{\mathbb{K}}$ consisting of common eigenfunctions for the $\eta$-action of $\mathbb{C}_{Y}[T]$ on $H_{0}^{\mathbb{K}}$. For $p \in \mathbb{C}[T]$ and $w \in W_{0}$ we have

$$
\eta(p(Y))(\gamma) \xi_{w}(\gamma)=\left(w^{-1} p\right)(\gamma) \xi_{w}(\gamma)
$$

as $H_{0}$-valued regular functions in $\gamma \in T$. 


\subsection{The cocycle values}

Write

$$
R_{i}(z ; \gamma)=c\left(z ; k_{i}\right)^{-1}\left(\eta\left(T_{i}\right)(\gamma)-b\left(z ; k_{i}\right)\right), \quad 0 \leq i \leq N
$$

viewed as a $\operatorname{End}\left(H_{0}\right)$-valued function that depends rationally on $z$ and rationally on $\gamma \in T$ for $i=0$ and is otherwise $\gamma$-independent.

\section{Lemma 4.3 (i) We have}

$$
\begin{aligned}
& C_{\left(s_{i}, e\right)}(t, \gamma)=R_{i}\left(t^{a_{i}^{\vee}} ; \gamma\right), \quad 0 \leq i \leq N, \\
& C_{(\omega, e)}(t, \gamma)=\eta(\omega)(\gamma), \quad \omega \in \Omega,
\end{aligned}
$$

and $C_{\iota}$ is the $\mathbb{K}$-linear extension of the involution of $H_{0}$ determined by

$$
C_{\iota}\left(T_{w}\right)=T_{w^{-1}}, \quad w \in W_{0} .
$$

(ii) $R_{i}(z ; \gamma) R_{i}\left(z^{-1} ; \gamma\right)=$ id for $0 \leq i \leq N$.

Remark 4.4 Note that

$$
C_{(e, w)}(t, \gamma)=C_{\iota} C_{(w, e)}\left(\gamma^{-1}, t^{-1}\right) C_{\iota}, \quad w \in W
$$

so part (i) of the previous lemma uniquely determines $C_{\mathrm{w}}$ for all $\mathrm{w} \in \mathbb{W}$.

\section{Solutions of $\mathrm{BqKZ}$}

The main result of this section is the construction of a particular meromorphic solution $\Phi$ of BqKZ called the basic asymptotically free solution. The idea is as follows. We first look for $v \in H_{0}$ and $G \in \mathbb{K}$ such that $G v$ will be the leading term of a solution of BqKZ in some asymptotic region. These are obtained by looking for a solution of an asymptotic version of $\mathrm{BqKZ}$, that is, $\mathrm{BqKZ}$ in which the $q$-connection matrices are replaced by their limit values in the asymptotic region.

Next, we gauge BqKZ by $G$ and look for a power series solution $\Psi$ of the gauged $\mathrm{BqKZ}$ equation converging deep inside the asymptotic region and which has constant term $v$. By meromorphic continuation, $\Psi$ can be extended to a meromorphic solution of the gauged BqKZ equation, yielding the desired solution $\Phi=G \Psi \in H_{0}^{\mathbb{K}}$ of BqKZ. Apart from the construction itself, we will derive various properties of $\Phi$ and give an explicit $\mathbb{F}$-basis of SOL, but we start with the computation of the leading term.

\subsection{The leading term}

In order to find these $v$ and $G$, we first need to compute the asymptotic leading terms of the $q$-connection matrices $C_{(\lambda, e)}(t, \gamma)\left(\lambda \in P^{\vee}\right)$ as $\left|t^{-\alpha_{i}^{\vee}}\right| \rightarrow 0(1 \leq i \leq N)$. 
Let $\mathcal{A} \subset \mathbb{C}[T \times\{1\}]=\mathbb{C}\left[x_{1}^{ \pm 1}, \ldots, x_{N}^{ \pm 1}\right] \subset \mathbb{C}[T \times T]$ be the subring $\mathcal{A}:=$ $\mathbb{C}\left[x^{-\alpha_{1}^{\vee}}, \ldots, x^{-\alpha_{N}^{\vee}}\right]$. Let $Q(\mathcal{A})$ denote its quotient field and write $Q_{0}(\mathcal{A})$ for the subring of $Q(\mathcal{A})$ consisting of rational functions which are regular at the point $x^{-\alpha_{i}^{\vee}}=0$ $(1 \leq i \leq N)$. We consider $Q_{0}(\mathcal{A}) \otimes \mathbb{C}[T]$ as subring of $\mathbb{C}(T \times T)$ in the natural way.

Lemma 5.1 Let $\lambda \in P^{\vee}$. We have

$$
C_{(\lambda, e)} \in\left(Q_{0}(\mathcal{A}) \otimes \mathbb{C}[T]\right) \otimes \operatorname{End}\left(H_{0}\right)
$$

If we write $C_{(\lambda, e)}^{(0)}=\left.C_{(\lambda, e)}\right|_{x^{-\alpha_{1}^{\vee}}=0, \ldots, x^{-\alpha_{N}} \vee 0} \in \mathbb{C}[T] \otimes \operatorname{End}\left(H_{0}\right)$, we have

$$
C_{(\lambda, e)}^{(0)}=\delta_{\underline{k}}^{\lambda} \eta\left(T_{w_{0}} Y^{w_{0}(\lambda)} T_{w_{0}}^{-1}\right) .
$$

Proof First we consider $\lambda \in P_{+}^{\vee}$. Suppose we have a reduced expression $\mathrm{t}(\lambda)=$ $s_{i_{1}} \ldots s_{i_{r}} \omega\left(0 \leq i_{1}, \ldots, i_{r} \leq N, \omega \in \Omega\right)$. Then

$$
C_{(\lambda, e)}(t, \gamma)=R_{i_{1}}\left(t^{a_{i_{1}}^{\vee}} ; \gamma\right) R_{i_{2}}\left(t^{s_{i_{1}}\left(a_{i_{2}}^{\vee}\right)} ; \gamma\right) \ldots R_{i_{r}}\left(t^{s_{i_{1}} \ldots s_{i_{r-1}}\left(a_{i_{r}}^{\vee}\right)} ; \gamma\right) \eta(\omega)(\gamma) .
$$

It follows that $C_{(\lambda, e)} \in(Q(\mathcal{A}) \otimes \mathbb{C}[T]) \otimes \operatorname{End}\left(H_{0}\right)$. Expanding $C_{(-\lambda, e)}$ along the reduced expression $\mathrm{t}(-\lambda)=\omega^{-1} s_{i_{r}} \ldots s_{i_{1}}$ gives an expression similar to (5.3), from which we conclude that also $C_{(-\lambda, e)} \in(Q(\mathcal{A}) \otimes \mathbb{C}[T]) \otimes \operatorname{End}\left(H_{0}\right)$. Since the $R_{i}(z ; \gamma)$ are analytic at $z=0$ and $z=\infty$, we have $C_{(\lambda, e)}, C_{(-\lambda, e)} \in\left(Q_{0}(\mathcal{A}) \otimes \mathbb{C}[T]\right) \otimes$ End $\left(H_{0}\right)$. Writing an arbitrary weight as the difference of two dominant weights and using the cocycle property we conclude (5.1) for any $\lambda \in P^{\vee}$.

To prove (5.2), we will first compute the limit of $C_{(\lambda, e)}(t, \gamma)$ as $\left|t^{\alpha_{i}^{\vee}}\right| \rightarrow 0$ for $1 \leq i \leq N$ and then use this together with the cocycle property to find $C_{(\lambda, e)}^{(0)}(\gamma)$, which is the limit as $\left|t^{-\alpha_{i}^{\vee}}\right| \rightarrow 0(1 \leq i \leq N)$. Similarly as in the proof of (5.1), it suffices to consider only dominant weights. Assume we have $\lambda \in P_{+}^{\vee}$ a reduced expression for $\mathrm{t}(\lambda)$ as above and put $u=s_{i_{1}} \ldots s_{i_{r}}$. By formulas (2.2.9) and (2.2.5) from [14] we have $\left\{a_{i_{1}}, s_{i_{1}}\left(a_{i_{2}}\right), \ldots, s_{i_{1}} \ldots s_{i_{r-1}}\left(a_{i_{r}}\right)\right\}=S\left(u^{-1}\right)=S\left(\omega^{-1} u^{-1}\right)=S(\mathrm{t}(-\lambda))$. Because $\lambda \in P_{+}^{\vee}$ we have

$$
S(\mathrm{t}(-\lambda))=\left\{\alpha+m c \mid \alpha \in R_{-}, 1 \leq m \leq-\langle\lambda, \alpha\rangle\right\}
$$

(cf. [14, §2.4]), and thus, since $w\left(a^{\vee}\right)=(w a)^{\vee}(a \in S, w \in W)$, we have $\left|t^{b^{\vee}}\right| \rightarrow \infty$ $(b \in S(\mathrm{t}(-\lambda)))$ as $\left|t^{\alpha_{i}^{\vee}}\right| \rightarrow 0(1 \leq i \leq N)$. Observe that $\lim _{z \rightarrow \infty} R_{i}(z ; \gamma)=$ $k_{i}^{-1} \eta\left(T_{i}\right)(\gamma)$ for $0 \leq i \leq N$. It follows that

$$
C_{(\lambda, e)}(t, \gamma) \rightarrow k_{i_{1}}^{-1} \ldots k_{i_{r}}^{-1} \eta\left(Y^{\lambda}\right)(\gamma)=k(\mathrm{t}(\lambda))^{-1} \eta\left(Y^{\lambda}\right)(\gamma)
$$

as $\left|t^{\alpha_{i}^{\vee}}\right| \rightarrow 0$ for all $1 \leq i \leq N$. More generally, we conclude that

$$
C_{(\lambda, e)}(t, \gamma) \rightarrow \delta_{\underline{k}}^{-\lambda} \eta\left(Y^{\lambda}\right)(\gamma), \quad \lambda \in P^{\vee}
$$


as $\left|t^{\alpha_{i}^{\vee}}\right| \rightarrow 0$ for all $1 \leq i \leq N$. In order to find $C_{(\lambda, e)}^{(0)}$, we use the cocycle property to write

$$
C_{(\lambda, e)}(t, \gamma)=C_{\left(w_{0}, e\right)}(t, \gamma) C_{\left(w_{0}(\lambda), e\right)}\left(w_{0} t, \gamma\right) C_{\left(w_{0}, e\right)}\left(q^{-w_{0}(\lambda)} w_{0} t, \gamma\right)
$$

and consider the limit as $\left|t^{-\alpha_{i}^{\vee}}\right| \rightarrow 0$ for $1 \leq i \leq N$. Note that $C_{\left(w_{0}, e\right)}(t, \gamma) \rightarrow$ $k\left(w_{0}\right)^{-1} \eta\left(T_{w_{0}}\right)$ as $\left|t^{-\alpha_{i}^{\vee}}\right| \rightarrow 0$ for $1 \leq i \leq N$. Hence, using (5.4),

$$
C_{(\lambda, e)}^{(0)}=\delta_{\underline{k}}^{-w_{0}(\lambda)} \eta\left(T_{w_{0}} Y^{w_{0}(\lambda)} T_{w_{0}}^{-1}\right)=\delta_{\underline{k}}^{\lambda} \eta\left(T_{w_{0}} Y^{w_{0}(\lambda)} T_{w_{0}}^{-1}\right)
$$

where the last equality follows from (2.3).

The previous lemma implies that the asymptotic form of the quantum KZ equations

$$
C_{(\lambda, e)}(t, \gamma) f\left(q^{-\lambda} t, \gamma\right)=f(t, \gamma), \quad \lambda \in P^{\vee}
$$

in the asymptotic region $\left|t^{\alpha_{i}^{\vee}}\right| \gg 0(1 \leq i \leq N)$ is

$$
\delta_{\underline{k}}^{\lambda} \eta\left(T_{w_{0}} Y^{w_{0}(\lambda)} T_{w_{0}}^{-1}\right)(\gamma) f\left(q^{-\lambda} t, \gamma\right)=f(t, \gamma), \quad \lambda \in P^{\vee} .
$$

Let $\theta_{q} \in \mathcal{O}(T)$ denote the theta function associated with the root system $R$ (see [11]), defined by

$$
\theta_{q}(t):=\sum_{\lambda \in P^{\vee}} q^{\frac{1}{2}\langle\lambda, \lambda\rangle} t^{\lambda}
$$

for $t \in T$. Note that $\theta_{q}$ is invariant under the action of $W_{0}$ on $\mathcal{O}(T)$. Furthermore, it satisfies $\theta_{q}\left(t^{-1}\right)=\theta_{q}(t)$ and

$$
\theta_{q}\left(q^{\mu} t\right)=q^{-\frac{1}{2}\langle\mu, \mu\rangle} t^{-\mu} \theta_{q}(t)
$$

for all $\mu \in P^{\vee}$.

Let $G \in \mathbb{K}$ be given by

$$
G(t, \gamma):=\frac{\theta_{q}\left(t w_{0}(\gamma)^{-1}\right)}{\theta_{q}\left(\delta_{\underline{k}} t\right) \theta_{q}\left(\delta_{\underline{k}}^{-1} w_{0}(\gamma)^{-1}\right)} .
$$

Proposition 5.2 We have:

(i) $\iota(G)=G$.

(ii) $G(t, \gamma)$ satisfies the $q$-difference equations 


$$
G\left(q^{-\lambda} t, q^{\mu} \gamma\right)=\delta_{\underline{k}}^{-\lambda-\mu} q^{-\left\langle w_{0}(\lambda), \mu\right\rangle} t^{w_{0}(\mu)} \gamma^{-w_{0}(\lambda)} G(t, \gamma)
$$

for $\lambda, \mu \in P^{\vee}$.

(iii) $f^{(0)}(t, \gamma):=G(t, \gamma) T_{w_{0}}$ is a solution of $(5.5)$ and $\tau(\iota) f^{(0)}=f^{(0)}$.

Proof By construction we have (i). From (5.7) it follows that $G$ satisfies $G\left(q^{-\lambda} t, \gamma\right)=$ $\delta_{k}^{-\lambda} \gamma^{-w_{0}(\lambda)} G(t, \gamma)$ for all $\lambda \in P^{\vee}$. In view of (i) this suffices to prove (ii). (iii) easily follows from (i) and (ii).

\subsection{The basic asymptotically free solution $\Phi$}

As indicated in the introduction of this section, we are now going to gauge BqKZ by $G$. We obtain the gauged $q$-connection matrices

$$
\begin{aligned}
D_{(\lambda, \mu)}(t, \gamma) & =G(t, \gamma)^{-1} C_{(\lambda, \mu)}(t, \gamma) G\left(q^{-\lambda} t, q^{\mu} \gamma\right) \\
& =\delta_{\underline{k}}^{-\lambda-\mu} q^{-\left\langle\mu, w_{0}(\lambda)\right\rangle} \gamma^{-w_{0}(\lambda)} t^{w_{0}(\mu)} C_{(\lambda, \mu)}(t, \gamma),
\end{aligned}
$$

for $\lambda, \mu \in P^{\vee}$. It is clear that for $f \in H_{0}^{\mathbb{K}}$, we have $f \in$ SOL if and only if $g:=G^{-1} f \in H_{0}^{\mathbb{K}}$ satisfies the holonomic system of $q$-difference equations

$$
D_{(\lambda, \mu)}(t, \gamma) g\left(q^{-\lambda} t, q^{\mu} \gamma\right)=g(t, \gamma), \quad \lambda, \mu \in P^{\vee}
$$

as $H_{0}$-valued meromorphic functions in $(t, \gamma) \in T \times T$.

We write $\mathcal{B}$ for the analog of $\mathcal{A}$ corresponding to the second copy of $T$ in $T \times T$. That is, $\mathcal{B}$ is the subring $\mathcal{B}:=\mathbb{C}\left[y^{\alpha_{1}^{\vee}}, \ldots, y^{\alpha_{N}^{\vee}}\right]$ of $\mathbb{C}[\{1\} \times T]=\mathbb{C}\left[y_{1}^{ \pm 1}, \ldots, y_{N}^{ \pm 1}\right]$. Similarly, we write $Q(\mathcal{B})$ for its quotient field and $Q_{0}(\mathcal{B})$ for the subring of $Q(\mathcal{B})$ consisting of rational functions which are regular at the point $y^{\alpha_{j}^{\vee}}=0(1 \leq j \leq N)$. We consider $Q_{0}(\mathcal{A}) \otimes \mathcal{B}$ and $\mathcal{A} \otimes Q_{0}(\mathcal{B})$ as subrings of $\mathbb{C}(T \times T)$ in the natural way.

In the proof of the lemma below, we will need a partial order $\succeq$ on $P^{\vee}$. First recall the dominance partial order $\geq$ on $P_{+}^{\vee}$, which is defined by

$$
\lambda \geq \mu \Longleftrightarrow \lambda-\mu \in Q_{+}^{\vee},
$$

for $\lambda, \mu \in P_{+}^{\vee}$. We can extend this to a partial order on $P^{\vee}$ as follows. For $\lambda \in P^{\vee}$ write $\lambda_{+}$for the unique dominant coweight in the orbit $W_{0} \lambda$ and let $\bar{v}_{\lambda}$ be the shortest $w \in W_{0}$ such that $w\left(\lambda_{+}\right)=\lambda$. For $\lambda, \mu \in P^{\vee}$, we say that $\lambda \succeq \mu$ if either

(i) $\lambda_{+}>\mu_{+}$, or

(ii) $\lambda_{+}=\mu_{+}$and $\bar{v}_{\lambda} \geq \bar{v}_{\mu}$ (in the Bruhat order).

Note that with respect to this order, the anti-dominant coweight $w_{0}\left(\lambda_{+}\right)$is the largest element in the orbit $W_{0} \lambda$. More details can be found in [14, §2.7].

The following lemma describes the asymptotic behavior of the gauged $q$-connection matrices. It allows us to put them in the context of the general theory of solutions of $q$-difference equations as described in the appendix of [18] and is therefore a key ingredient in the construction of $\Phi$. 
Lemma 5.3 Set $A_{i}=D_{\left(\varpi_{i}^{\vee}, e\right)}$ and $B_{i}=D_{\left(e, \varpi_{i}^{\vee}\right)}$ for $1 \leq i \leq N$.

(i) $A_{i} \in\left(Q_{0}(\mathcal{A}) \otimes \mathcal{B}\right) \otimes \operatorname{End}\left(H_{0}\right)$ and $B_{j} \in\left(\mathcal{A} \otimes Q_{0}(\mathcal{B})\right) \otimes \operatorname{End}\left(H_{0}\right)$.

(ii) Write $A_{i}^{(0,0)} \in \operatorname{End}\left(H_{0}\right)$ and $B_{i}^{(0,0)} \in \operatorname{End}\left(H_{0}\right)$ for the value of $A_{i}$ and $B_{i}$ at $x^{-\alpha_{r}^{\vee}}=0=y^{\alpha_{s}^{\vee}}(1 \leq r, s \leq N)$. For $w \in W_{0}$ we have

$$
A_{i}^{(0,0)}\left(T_{w_{0}} T_{w}\right)= \begin{cases}0 & \text { if } w^{-1} w_{0}\left(\varpi_{i}^{\vee}\right) \neq w_{0}\left(\varpi_{i}^{\vee}\right), \\ T_{w_{0}} T_{w} & \text { if } w^{-1} w_{0}\left(\varpi_{i}^{\vee}\right)=w_{0}\left(\varpi_{i}^{\vee}\right)\end{cases}
$$

and

$$
B_{i}^{(0,0)}\left(T_{w_{0}} T_{w}\right)= \begin{cases}0 & \text { if } w\left(\varpi_{i}^{\vee}\right) \neq \varpi_{i}^{\vee} \\ T_{w_{0}} T_{w} & \text { if } w\left(\varpi_{i}^{\vee}\right)=\varpi_{i}^{\vee}\end{cases}
$$

Proof We give the proof of (i), which differs substantially from the $\mathrm{GL}_{N}$ case (cf. [18, Lem. 5.2]), and omit the proof of (ii), which is similar. By (5.10) we have

$$
A_{i}(t, \gamma)=\delta_{\underline{k}}^{-\varpi_{i}^{\vee}} \gamma^{-w_{0}\left(\varpi_{i}^{\vee}\right)} C_{\left(\varpi_{i}^{\vee}, e\right)}(t, \gamma)
$$

Because of (5.1), we only need to worry about the $\gamma$-dependence of $A_{i}(t, \gamma)$.

Let $\mathrm{t}\left(\varpi_{i}^{\vee}\right)=\omega s_{i_{1}} \ldots s_{i_{r}}\left(\omega \in \Omega, 0 \leq i_{1}, \ldots, i_{r} \leq N\right)$ be a reduced expression. Then, in view of the cocycle condition, Lemma 4.3 and formula (4.5),

$$
C_{\left(\varpi_{i}^{\vee}, e\right)}(t, \gamma)=\eta(\omega)(\gamma) C_{\left(s_{i_{1}} \ldots s_{i}, e\right)}\left(\omega^{-1} t, \gamma\right)=\sum_{w \leq t\left(\varpi_{i}^{\vee}\right)} a_{w}(t) \eta\left(T_{w}\right)(\gamma)
$$

for certain $a_{w} \in Q_{0}(\mathcal{A})$. Now consider such $w \in W$ with $w \leq \mathrm{t}\left(\varpi_{i}^{\vee}\right)$. We have a unique decomposition $w=\mathrm{t}(\lambda) \widetilde{w}$, with $\lambda=w(0) \in P^{\vee}$ and $\widetilde{w} \in W_{0}$. Then,

$$
\mathrm{t}(\lambda)=\mathrm{t}\left(\bar{v}_{\lambda}\left(\lambda_{+}\right)\right)=\bar{v}_{\lambda} \mathrm{t}\left(\lambda_{+}\right) \bar{v}_{\lambda}^{-1}
$$

hence $w=\bar{v}_{\lambda} \mathrm{t}\left(\lambda_{+}\right) \bar{v}_{\lambda}^{-1} \widetilde{w}$. Multiple use of [14, (3.1.7)] yields $T_{w}=h T_{\mathrm{t}\left(\lambda_{+}\right)} h^{\prime}=$ $h Y^{\lambda+} h^{\prime}$ for some $h, h^{\prime} \in H_{0}$, hence

$$
\eta\left(T_{w}\right)(\gamma)=\eta(h) \eta\left(Y^{\lambda_{+}}\right)(\gamma) \eta\left(h^{\prime}\right) .
$$

It remains to show that $\gamma^{-w_{0}\left(\varpi_{i}^{\vee}\right)} \eta\left(Y^{\lambda_{+}}\right)(\gamma) \in \mathcal{B} \otimes \operatorname{End}\left(H_{0}\right)$. We can use (4.3) to write

$$
\eta\left(Y^{\lambda_{+}}\right)(\gamma) T_{w}=\sum_{u \leq w} p_{u, w}^{\lambda_{+}}(\gamma) T_{u}
$$

with $p_{u, w}^{\lambda_{+}}(\gamma) \in \operatorname{span}_{\mathbb{C}}\left\{\gamma^{\mu}\right\}_{\mu \in \Sigma\left(\lambda_{+}\right)}$and $p_{w, w}^{\lambda_{+}}(\gamma)=\gamma^{w^{-1}\left(\lambda_{+}\right)}$. Thus, we need to show that

$$
\gamma^{-w_{0}\left(\varpi_{+}\right)+\mu} \in \mathcal{B} \quad \forall \mu \in \Sigma\left(\lambda_{+}\right),
$$


i.e., that $-w_{0}\left(\varpi_{i}^{\vee}\right)+\mu \in Q_{+}^{\vee}$ for all $\mu \in \Sigma\left(\lambda_{+}\right)$. Since $\Sigma\left(\lambda_{+}\right)$is $W_{0}$-invariant and $w_{0}\left(Q_{+}^{\vee}\right)=-Q_{+}^{\vee}$, this is equivalent to showing that $-\varpi_{i}^{\vee}+\mu \in-Q_{+}^{\vee}$ for all $\mu \in \Sigma\left(\lambda_{+}\right)$, or

$$
\varpi_{i}^{\vee}-\mu \in Q_{+}^{\vee} \quad \forall \mu \in \Sigma\left(\lambda_{+}\right) .
$$

Now the fact that $w \leq \mathrm{t}\left(\varpi_{i}^{\vee}\right)$ in the Bruhat order on $W$ implies that $\lambda \preceq \varpi_{i}^{\vee}$ (cf. [14, (2.7.11)]), and hence either $\lambda_{+}=\varpi_{i}^{\vee}$ or $\lambda_{+}<\varpi_{i}^{\vee}$. Fix $\mu \in \Sigma\left(\lambda_{+}\right)$. In the first case, if $\lambda_{+}=\varpi_{i}^{\vee}$, we have $\mu \in \varpi_{i}^{\vee}-Q_{+}^{\vee}$, since

$$
\Sigma\left(\varpi_{i}^{\vee}\right)=\bigcap_{v \in W_{0}} v\left(\varpi_{i}^{\vee}-Q_{+}^{\vee}\right)
$$

by [14, (2.6.2)]. Hence, $\varpi_{i}^{\vee}-\mu \in Q_{+}^{\vee}$. In the second case, if $\lambda_{+}<\varpi_{i}^{\vee}$, then $\Sigma\left(\lambda_{+}\right) \subset \Sigma\left(\varpi_{i}^{\vee}\right)$ by [14, (2.6.3)], and again $\mu \in \varpi_{i}^{\vee}-Q_{+}^{\vee}$. This concludes the proof for $A_{i}$. For $B_{i}$, use that $C_{\left(e, \varpi_{i}^{\vee}\right)}(t, \gamma)=C_{\iota} C_{\left(\varpi_{i}^{\vee}, e\right)}\left(\gamma^{-1}, t^{-1}\right) C_{\iota}$.

Part (ii) of the previous lemma asserts that the endomorphisms $A_{i}^{(0,0)}$ and $B_{i}^{(0,0)}$ are semisimple. Similarly as for $\mathrm{GL}_{N}$, the main theorem follows from the lemma together with the general theory of solutions of $q$-difference equations as described in the appendix of [18] (in particular [18, Thm. 8.6]).

For $\epsilon>0$, put $B_{\epsilon}:=\left\{t \in T|| t^{\alpha_{i}^{\vee}} \mid<\epsilon\right.$ for $\left.1 \leq i \leq N\right\}$ and $B_{\epsilon}^{-1}:=\{t \in T \mid$ $\left.t^{-1} \in B_{\epsilon}\right\}$.

Theorem 5.4 There exists a unique solution $\Psi \in H_{0}^{\mathbb{K}}$ of the gauged equations (5.11) such that, for some $\epsilon>0$,

(i) $\Psi(t, \gamma)$ admits an $H_{0}$-valued power series expansion

$$
\Psi(t, \gamma)=\sum_{\alpha, \beta \in Q_{+}^{\vee}} K_{\alpha, \beta} t^{-\alpha} \gamma^{\beta}, \quad\left(K_{\alpha, \beta} \in H_{0}\right)
$$

for $(t, \gamma) \in B_{\epsilon}^{-1} \times B_{\epsilon}$ which is normally convergent on compacta of $B_{\epsilon}^{-1} \times B_{\epsilon}$. In particular, $\Psi(t, \gamma)$ is analytic at $(t, \gamma) \in B_{\epsilon}^{-1} \times B_{\epsilon}$;

(ii) $K_{0,0}=T_{w_{0}}$.

Proof We only remark that in order to match the present situation with the one considered in [18, §8], one should take in [18, §8]: $M=2 N, A_{i}=A_{i}^{(0,0)}, A_{N+i}=B_{i}^{(0,0)}$ and $q_{i}=q^{2 /\left\|\alpha_{i}\right\|^{2}}$ for $1 \leq i \leq N$ and variables $z_{i}=x^{-\alpha_{i}^{\vee}}$ and $z_{N+j}=y^{\alpha_{j}^{\vee}}$ for $1 \leq i, j \leq N$.

Definition 5.5 We call $\Phi:=G \Psi \in$ SOL the basic asymptotically free solution of $\mathrm{BqKZ}$.

The $\tau(\iota)$-invariance of SOL, the $\iota$-invariance of $G$ and the uniqueness part of Theorem 5.4 imply that $\Phi$ enjoys the following duality property. 
Theorem 5.6 (Duality) The basic asymptotically free solution $\Phi$ of BqKZ is self-dual, in the sense that

$$
\tau(\iota) \Phi=\Phi
$$

\subsection{Singularities}

In this subsection, we have a closer look at the analytic properties of $\Psi$. Write $q_{\alpha}:=$ $q^{2 /\|\alpha\|^{2}}$ for $\alpha \in R$ and set

$$
\mathcal{S}_{+}:=\left\{t \in T \mid t^{\alpha^{\vee}} \in k_{\alpha}^{-2} q_{\alpha}^{-\mathbb{N}} \text { for some } \alpha \in R_{+}\right\}
$$

Proposition 5.7 The $H_{0}$-valued meromorphic function $\Psi$ is analytic on $T \backslash \mathcal{S}_{+}^{-1} \times$ $T \backslash \mathcal{S}_{+}$.

Proof Let $\lambda, \mu \in P_{+}^{\vee}$. By (5.10) and the cocycle property, $D_{(\lambda, \mu)}(t, \gamma)$ is regular at $(t, \gamma)=(s, \zeta)$ if $C_{\left(\varpi_{i}^{\vee}, \varpi_{j}^{\vee}\right)}\left(q^{-v} t, q^{\xi} \gamma\right)$ is regular at $(t, \gamma)=(s, \zeta)$ for all $1 \leq$ $i, j \leq N$ and $\xi, v \in P_{+}^{\vee}$. This in turn holds, again by virtue of the cocycle property together with (5.1), if $C_{\left(\omega_{j}^{\vee}, e\right)}\left(q^{-v} t, \gamma\right)$ is regular at $(t, \gamma)=(s, \zeta)$ for all $v \in P_{+}^{\vee}$ and $1 \leq j \leq N$.

Suppose we have a reduced expression $\mathrm{t}\left(\varpi_{j}^{\vee}\right)=s_{i_{1}} \ldots s_{i_{r}} \omega(1 \leq j \leq N)$. Similarly as in the proof of Lemma 5.1, we have

$$
\left.C_{(\varpi)}^{\vee}, e\right)(t, \gamma)=R_{i_{1}}\left(t^{a_{i_{1}}^{\vee}} ; \gamma\right) \ldots R_{i_{r}}\left(t^{s_{i_{1}} \ldots s_{i_{r-1}}}\left(a_{i_{r}}^{\vee}\right) ; \gamma\right) \eta(\omega)(\gamma)
$$

and

$$
\begin{aligned}
\left\{a_{i_{1}}, s_{i_{1}}\left(a_{i_{2}}\right), \ldots, s_{i_{1}} \ldots s_{i_{r-1}}\left(a_{i_{r}}\right)\right\} & =S\left(\mathrm{t}\left(-\varpi_{j}^{\vee}\right)\right) \\
& =\left\{\alpha+m c \mid \alpha \in R_{-}, 1 \leq m \leq-\left\langle\varpi_{j}^{\vee}, \alpha\right\rangle\right\}
\end{aligned}
$$

Now $R_{i}(z ; \gamma)$ has only a simple pole at $z=k_{i}^{-2}$, so $C_{\left(\varpi_{j}^{\vee}, e\right)}(t, \gamma)$ has possibly poles at

$$
t^{a^{\vee}}=k_{a}^{-2}, \quad a \in S\left(\mathrm{t}\left(-\varpi_{j}^{\vee}\right)\right)
$$

Note that

$$
t^{(\alpha+m c)^{\vee}}=t^{\alpha^{\vee}+\left(2 m /\|\alpha\|^{2}\right) c}=q_{\alpha}^{m} t^{\alpha^{\vee}},
$$

hence there are possibly poles at

$$
q_{\alpha}^{m} t^{\alpha^{\vee}}=k_{\alpha}^{-2}, \quad \alpha \in R_{-}, 1 \leq m \leq-\left\langle\varpi_{j}^{\vee}, \alpha\right\rangle,
$$


or, equivalently, at

$$
t^{-\alpha^{\vee}}=q_{\alpha}^{-m} k_{\alpha}^{-2}, \quad \alpha \in R_{+}, 1 \leq m \leq\left\langle\varpi_{j}^{\vee}, \alpha\right\rangle .
$$

Consequently, $C_{\left(\varpi_{j}^{\vee}, e\right)}\left(q^{-v} t, \gamma\right)$ is regular at $t \in T \backslash \mathcal{S}_{+}^{-1}$ for all $v \in P_{+}^{\vee}$. By the considerations in the previous paragraph, we conclude that $D_{(\lambda, \mu)}(t, \gamma)$ is regular at $(t, \gamma) \in T \backslash \mathcal{S}_{+}^{-1} \times T \backslash \mathcal{S}_{+}$for all $\lambda, \mu \in P_{+}^{\vee}$.

Let $U \times V$ be a relatively compact open subset of $T \backslash \mathcal{S}_{+}^{-1} \times T \backslash \mathcal{S}_{+}$. Choose $\lambda, \mu \in P_{+}^{\vee}$ such that the closure of $q^{-\lambda} U \times q^{\mu} V$ is contained in $B_{\epsilon}^{-1} \times B_{\epsilon}$. Then as meromorphic $H_{0}$-valued function in $(t, \gamma) \in U \times V$, we have

$$
\Psi(t, \gamma)=D_{(\lambda, \mu)}(t, \gamma) \Psi\left(q^{-\lambda} t, q^{\mu} \gamma\right)
$$

and by Theorem 5.4(i) the proof is now complete.

Remark 5.8 The previous proposition gives, in particular, information about the singularities of the basic asymptotic solution $\Phi=G \Psi$. Unfortunately, it is not possible to precisely pinpoint the singularities of $G$. To overcome this issue, we could choose a different theta function in the definition of $G$, namely one for which we have a product formula available. The price we pay is that we have to enlarge the torus $T$. Let $\vartheta_{q} \in \mathcal{M}(T)$ denote the renormalized Jacobi theta function

$$
\vartheta_{q}(z):=\prod_{m \geq 0}\left(1-q^{m} z\right)\left(1-q^{m+1} / z\right)
$$

for $z \in \mathbb{C}^{\times}$. It satisfies

$$
\vartheta_{q}\left(q^{m} z\right)=(-z)^{-m} q^{-\frac{1}{2} m(m-1)} \vartheta_{q}(z), \quad m \in \mathbb{Z}
$$

Let $e \in \mathbb{N}$ be the unique positive integer such that $e\left\langle P^{\vee}, P^{\vee}\right\rangle=\mathbb{Z}$. For all $a \in S$, fix $k_{a}^{1 / 6 e}$ such that $k_{a}^{1 / 6 e}=k_{w(a)}^{1 / 6 e}$ for all $w \in W$. Now put $T^{\prime}:=\operatorname{Hom}_{\mathbb{Z}}\left(\frac{1}{6 e} P^{\vee}, \mathbb{C}^{\times}\right)$. The canonical map $T^{\prime} \rightarrow T$ gives rise to an embedding $\mathcal{M}(T \times T) \hookrightarrow \mathcal{M}\left(T^{\prime} \times T^{\prime}\right)$. Now define $\widehat{G} \in \mathcal{M}\left(T^{\prime} \times T^{\prime}\right)$ by

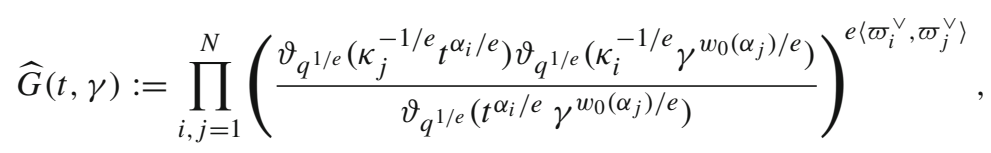

where $\kappa_{j}^{1 / e}:=\prod_{\alpha \in R_{+}} k_{\alpha}^{\left\langle\alpha_{j}, \alpha\right\rangle / e}$. Then, $\widehat{G}$ satisfies the properties of Proposition 5.2.

Corollary 5.9 (i) Write $\Psi(t, \gamma)=\sum_{\alpha \in Q_{+}^{\vee}} \Gamma_{\alpha}(\gamma) t^{-\alpha}$ for $(t, \gamma) \in B_{\epsilon}^{-1} \times B_{\epsilon}$, with $\Gamma_{\alpha}\left(\alpha \in Q_{+}^{\vee}\right)$ the analytic $H_{0}$-valued function $\Gamma_{\alpha}(\gamma):=\sum_{\beta \in Q_{+}^{\vee}} K_{\alpha, \beta} \gamma^{\beta}$ 
on $B_{\epsilon}$. Then each $\Gamma_{\alpha}$ can uniquely be extended to a meromorphic $H_{0}$-valued function on $T$, analytic on $T \backslash \mathcal{S}_{+}$, such that for $(t, \gamma) \in B_{\epsilon}^{-1} \times T \backslash \mathcal{S}_{+}$

$$
\Psi(t, \gamma)=\sum_{\alpha \in Q_{+}^{\vee}} \Gamma_{\alpha}(\gamma) t^{-\alpha}
$$

converging normally on compacta of $B_{\epsilon}^{-1} \times T \backslash \mathcal{S}_{+}$.

(ii) The leading term $\Gamma_{0}$ satisfies

$$
\Gamma_{0}(\gamma)=K(\gamma) T_{w_{0}}
$$

for some $K \in \mathcal{M}(T)$.

Proof (i) See [18, Lemma 5.7].

(ii) This is also similar as in [18], but for the convenience of the reader we provide the details. $\Psi$ satisfies $A_{i}(t, \gamma) \Psi\left(q^{-\varpi_{i}^{\vee}} t, \gamma\right)=\Psi(t, \gamma)$ for $1 \leq i \leq N$. Considering the limit $\left|t^{-\alpha_{j}^{\vee}}\right| \rightarrow 0$, we obtain

$$
\gamma^{-w_{0}\left(\varpi_{i}^{\vee}\right)} \eta\left(T_{w_{0}} Y^{w_{0}\left(\varpi_{i}^{\vee}\right)} T_{w_{0}}^{-1}\right)(\gamma) \Gamma_{0}(\gamma)=\Gamma_{0}(\gamma)
$$

for $1 \leq i \leq N$, and in view of Lemma 4.2 this forces

$$
\Gamma_{0}(\gamma)=K(\gamma) \eta\left(T_{w_{0}}\right) \xi_{e}(\gamma)=K(\gamma) T_{w_{0}}
$$

for some $K \in \mathcal{M}(T)$.

Remark 5.10 In the following section, we will give an explicit formula for $K(\gamma)$. It will follow immediately from an explicit formula for the leading term of the so-called Harish-Chandra series solution of a bispectral problem corresponding to BqKZ. In [18], for $\mathrm{GL}_{N}$, it is exactly the way around. There, the latter is found as a consequence of an explicit formula for $K(\gamma)$, which in turn is due to rather explicit expressions for the $q$-connection matrices of $\mathrm{BqKZ}$.

From Proposition 5.7 and its corollary, we obtain the following result for specialized spectral parameter.

Corollary 5.11 Fix $\zeta \in T \backslash \mathcal{S}_{+}$.

(i) The $H_{0}$-valued meromorphic function $\Psi(t, \gamma)$ in $(t, \gamma) \in T \times T$ can be specialized at $\gamma=\zeta$, giving rise to a meromorphic $H_{0}$-valued function $\Psi(t, \zeta)$ in $t \in T$, which is regular at $t \in T \backslash \mathcal{S}_{+}^{-1}$.

(ii) For $t \in B_{\epsilon}^{-1}$ we have the power series expansion

$$
\Psi(t, \zeta)=\sum_{\alpha \in Q_{+}^{\vee}} \Gamma_{\alpha}(\zeta) t^{-\alpha},
$$

converging normally on compacta of $B_{\epsilon}^{-1}$. 
(iii) $\Psi(t, \zeta)$ satisfies the system of $q$-difference equations

$$
D_{(\lambda, e)}(t, \zeta) \Psi\left(q^{-\lambda} t, \zeta\right)=\Psi(t, \zeta), \quad \forall \lambda \in P^{\vee}
$$

\subsection{Consistency}

$\mathrm{BqKZ}$ is a holonomic system of first-order $q$-difference equations with connection matrices depending rationally on $(t, \gamma) \in T \times T$, and therefore it is consistent (see $\left[6\right.$, Prop. 5.2]). This means that $\operatorname{dim}_{\mathbb{F}}(\mathrm{SOL})=\operatorname{dim}_{\mathbb{C}}\left(H_{0}\right)$, or, equivalently, that BqKZ allows a so-called fundamental matrix solution $U$. In [6], such a fundamental matrix solution was found by algebraic geometric arguments. A different approach, using the asymptotic solution $\Phi$, was taken in [18]. Here, we shortly repeat this latter approach for arbitrary root systems. The advantage of this approach is that it produces a basis of SOL in terms of asymptotically free solutions. For details, we refer to [18, §5.6].

We say that $F \in \operatorname{End}\left(H_{0}\right)^{\mathbb{K}}=\mathbb{K} \otimes \operatorname{End}\left(H_{0}\right)$ is an $\operatorname{End}\left(H_{0}\right)$-valued solution of $\mathrm{BqKZ}$, if

$$
C_{(\lambda, \mu)}(t, \gamma) F\left(q^{-\lambda} t, q^{\mu} \gamma\right)=F(t, \gamma), \quad \lambda, \mu \in P^{\vee},
$$

as $\operatorname{End}\left(H_{0}\right)$-valued meromorphic functions in $(t, \gamma) \in T \times T$.

Define $U \in \operatorname{End}\left(H_{0}\right)^{\mathbb{K}}$ by

$$
U\left(k(w)^{-1} T_{w_{0}} T_{w^{-1}}\right):=\tau(e, w) \Phi
$$

for $w \in W_{0}$.

Proposition 5.12 We have

(i) $U \in \operatorname{End}\left(H_{0}\right)^{\mathbb{K}}$ is an invertible solution of $B q K Z$ with values in $\operatorname{End}\left(H_{0}\right)$. In particular, identifying $\operatorname{End}\left(H_{0}\right)^{\mathbb{K}} \simeq \operatorname{End}_{\mathbb{K}}\left(H_{0}^{\mathbb{K}}\right)$ as $\mathbb{K}$-algebras, we have $U \in \mathrm{GL}_{\mathbb{K}}\left(H_{0}^{\mathbb{K}}\right)$.

(ii) $U^{\prime} \in \operatorname{End}\left(H_{0}\right)^{\mathbb{K}}$ is an $\operatorname{End}\left(H_{0}\right)$-valued meromorphic solution of $B q K Z$ if and only if $U^{\prime}=U F$ for some $F \in \operatorname{End}\left(H_{0}\right)^{\mathbb{F}}$.

(iii) $U$, viewed as $\mathbb{K}$-linear endomorphism of $H_{0}^{\mathbb{K}}$, restricts to an $\mathbb{F}$-linear isomorphism $U: H_{0}^{\mathbb{F}} \rightarrow$ SOL.

(iv) $\{\tau(e, w) \Phi\}_{w \in W_{0}}$ is an $\mathbb{F}$-basis of SOL.

Remark 5.13 The quantum KZ equations (3.11) form a consistent system of $q$-difference equations as well. For generic $\zeta \in T$ (that is, for $\zeta \in T$ where $\Phi(t, \gamma)$ can be specialized in $\gamma=\zeta$ and moreover $U(\cdot, \zeta)$ is invertible), this follows along the same line as above, but of course one can use [6, Prop. 5.2] again, which applies for all $\zeta \in T$. 


\section{Correspondence with bispectral problems}

For the principal series representation $M_{\zeta}$ ( $\zeta$ generic) of $H$, Cherednik [2, Thm. 3.4] constructed a map that embeds the associated solution space of the quantum affine $\mathrm{KZ}$ equation (3.2) into the solution space of a system of $q$-difference equations, involving the Macdonald $q$-difference operator. This is a special case of a correspondence between the solutions of the quantum affine KZ equations associated with an arbitrary finite-dimensional $H$-module $M$ and a more general system of $q$-difference equations (see [3]).

We will consider the map when $M$ is the formal principal series module $M=$ $\operatorname{Ind}_{\mathbb{C}_{Y}[T]}^{H}\left(\mathbb{C}_{Y}[T]\right)$ (see Sect. 4.1). In this case, Cherednik's correspondence yields an embedding $\chi_{+}$of SOL into the solution space of a bispectral problem for the Macdonald $q$-difference operators.

\subsection{The bispectral problem for the Macdonald $q$-difference operators}

Using the action of $\mathbb{W}$ on $\mathbb{C}(T \times T)$ given by (3.5), we can form the smash product algebra $\mathbb{C}(T \times T) \# \mathbb{W}$. It contains $\mathbb{C}(T) \#_{q} W \simeq \mathbb{C}(T \times\{1\}) \#(W \times\{e\})$ and $\mathbb{C}(T) \#_{q^{-1}} W \simeq \mathbb{C}(\{1\} \times T) \#(\{e\} \times W)$ as subalgebras. In this interpretation, Cherednik's algebra homomorphism $\rho_{\underline{k}^{-1}, q}: H\left(\underline{k}^{-1}\right) \rightarrow \mathbb{C}(T) \#_{q} W$ (see Theorem 2.8) gives rise to an algebra homomorphism

$$
\rho_{\underline{k}^{-1}, q}^{x}: H\left(\underline{k}^{-1}\right) \rightarrow \mathbb{C}(T \times T) \# \mathbb{W},
$$

considered as $q$-difference reflection operators in the first torus variable, and similarly $\rho_{\underline{k}, q^{-1}}: H(\underline{k}) \rightarrow \mathbb{C}(T) \#_{q^{-1}} W$ to an algebra homomorphism

$$
\rho_{\underline{k}, q^{-1}}^{y}: H(\underline{k}) \rightarrow \mathbb{C}(T \times T) \# \mathbb{W},
$$

considered as $q$-difference reflection operators in the second torus variable. Note that the images of $\rho_{\underline{k}^{-1}, q}^{x}$ and $\rho_{\underline{k}, q^{-1}}^{y}$ in $\mathbb{C}(T \times T) \# \mathbb{W}$ commute, so we can form the algebra homomorphism

$$
\rho_{\underline{k}^{-1}, q}^{x} \otimes \rho_{\underline{k}, q^{-1}}^{y}: H\left(\underline{k}^{-1}\right) \otimes H(\underline{k}) \rightarrow \mathbb{C}(T \times T) \# \mathbb{W} .
$$

The maps $\rho_{\underline{k}^{-1}, q}^{x}$ and $\rho_{\underline{k}, q^{-1}}^{y}$ are related as follows.

Lemma 6.1 Let $^{\circ}: H\left(\underline{k}^{-1}\right) \rightarrow H(\underline{k})$ be defined as the unique algebra isomorphism satisfying

$$
T_{i}^{\circ}=T_{i}^{-1}, \quad \omega^{\circ}=\omega,
$$

for $0 \leq i \leq N$ and $\omega \in \Omega$. Then, we have

$$
\rho_{\underline{k}, q^{-1}}^{y}\left(h^{\circ}\right)=\iota \rho_{\underline{k}^{-1}, q}^{x}(h) \iota
$$

for all $h \in H\left(\underline{k}^{-1}\right)$. 
Proof Since $\rho_{\underline{k}^{-1}, q}^{x}, \rho_{\underline{k}, q^{-1}}^{y}$ and ${ }^{\circ}$ are algebra homomorphisms, the lemma follows by verifying (6.1) for $T_{i}(0 \leq i \leq N)$ and $\omega \in \Omega$. Let $0 \leq i \leq N$ and $f \in \mathbb{K}$. In $H(\underline{k})$, we have $T_{i}^{-1}=T_{i}+k_{i}^{-1}-k_{i}$, hence, on the one hand,

$$
\left(\rho_{\underline{k}, q^{-1}}^{y}\left(T_{i}^{-1}\right) f\right)(t, \gamma)=k_{i}^{-1} f(t, \gamma)+c_{a_{i} ; \underline{k}, q^{-1}}(\gamma)\left(f\left(t, s_{i}^{\diamond} \gamma\right)-f(t, \gamma)\right) .
$$

On the other hand,

$$
\begin{aligned}
& \left(\iota \rho_{\underline{k}^{-1}, q}^{x}\left(T_{i}\right) \iota f\right)(t, \gamma)=\left(\rho_{\underline{k}^{-1}, q}^{x}\left(T_{i}\right) \iota f\right)\left(\gamma^{-1}, t^{-1}\right) \\
& =k_{i}^{-1}(\iota f)\left(\gamma^{-1}, t^{-1}\right)+c_{a_{i} ; \underline{k}^{-1}, q}\left(\gamma^{-1}\right)\left((\iota f)\left(s_{i} \gamma^{-1}, t^{-1}\right)-(\iota f)\left(\gamma^{-1}, t^{-1}\right)\right) \\
& =k_{i}^{-1} f(t, \gamma)+c_{a_{i} ; \underline{k}, q^{-1}}(\gamma)\left(f\left(t, s_{i}^{\diamond} \gamma\right)-f(t, \gamma)\right),
\end{aligned}
$$

where we used (2.4) for the last equality. The verification for $\omega \in \Omega$ is easier and left to the reader.

By means of the canonical action of $\mathbb{C}(T \times T) \# \mathbb{W}$ on $\mathbb{C}(T \times T)$, the subalgebra $\mathbb{D}:=\mathbb{C}(T \times T) \#\left(P^{\vee} \times P^{\vee}\right) \subset \mathbb{C}(T \times T) \# \mathbb{W}$ can be identified with the algebra of $q$-difference operators on $T \times T$ with rational coefficients. Any element $D \in \mathbb{C}(T \times$ $T) \# \mathbb{W}$ has an expansion

$$
D=\sum_{\mathrm{w} \in \mathbb{W}_{0}} D_{\mathrm{w}} \mathrm{w},
$$

with $D_{\mathrm{w}} \in \mathbb{D}$. Since this expansion is unique, we have a well-defined $\mathbb{C}(T \times T)$-linear map Res: $\mathbb{C}(T \times T) \# \mathbb{W} \rightarrow \mathbb{D}$, determined by

$$
\operatorname{Res}(D):=\sum_{\mathrm{w} \in \mathbb{W}_{0}} D_{\mathrm{w}},
$$

with $D \in \mathbb{C}(T \times T) \# \mathbb{W}$ given as in (6.2). Let $\mathbb{C}(T \times T)^{\mathbb{W}_{0}}$ denote the field of $\mathbb{W}_{0^{-}}$ invariant rational functions on $T \times T$. Restricted to $\mathbb{C}(T \times T)^{\mathbb{W}_{0}}$, we have $\left.D\right|_{\mathbb{C}(T \times T)} \mathbb{W}_{0}=\left.\operatorname{Res}(D)\right|_{\mathbb{C}(T \times T)} \mathbb{W}_{0}$ for all $D \in \mathbb{C}(T \times T) \# \mathbb{W}$.

It is well known (see e.g., [14, (4.2.10)]) that the center $Z(H)$ of the affine Hecke algebra $H$ is $\mathbb{C}_{Y}[T]^{W_{0}}$. For $p \in \mathbb{C}[T]^{W_{0}}$, set

$$
L_{p}^{x}:=\operatorname{Res}\left(\rho_{\underline{k}^{-1}, q}^{x}(p(Y))\right) \in \mathbb{D}
$$

where $p(Y)$ is considered as element of $Z\left(H\left(\underline{k}^{-1}\right)\right)$, and set

$$
L_{p}^{y}:=\operatorname{Res}\left(\rho_{\underline{k}, q^{-1}}^{y}(p(Y))\right) \in \mathbb{D}
$$

where $p(Y)$ is considered as element of $Z(H(\underline{k}))$. It is well known that the operators $L_{p}^{x}$ (and hence $\left.L_{p}^{y}\right)$ are pairwise commuting and $\left(W_{0} \times W_{0}\right)$-invariant, and by 
construction $\left[L_{p}^{x}, L_{p^{\prime}}^{y}\right]=0$ in $\mathbb{D}$ for all $p, p^{\prime} \in \mathbb{C}[T]^{W_{0}}$. The operators $L_{p}^{x}$ and $L_{p}^{y}$ are related as follows.

Lemma 6.2 For $p \in \mathbb{C}[T]^{W_{0}}$, we have

$$
L_{p}^{y}=\iota L_{p}^{x} \iota
$$

Proof Similarly as for $\mathrm{GL}_{N}$ (see $[18, \S 6.2]$ ), the lemma follows from (6.1) together with the fact that

$$
p(Y)^{\circ}=p(Y), \quad p \in \mathbb{C}[T]^{W_{0}}
$$

with $^{\circ}: H\left(\underline{k}^{-1}\right) \rightarrow H(\underline{k})$ as defined in Lemma 6.1. We elaborate on the proof of (6.4), which is different than for $\mathrm{GL}_{N}$. Note that since $p \in \mathbb{C}[T]^{W_{0}}$, the result follows if we can prove that $\left(Y^{\lambda}\right)^{\circ}=T_{w_{0}} Y^{w_{0}(\lambda)} T_{w_{0}}^{-1}$ for $\lambda \in P^{\vee}$. Moreover, it suffices to show this only for specific elements of $P^{\vee}$, as we demonstrate first. For any $\lambda \in P^{\vee}$, let $v_{\lambda}$ be the shortest element of $W_{0}$ such that $v_{\lambda}(\lambda)=w_{0}(\lambda)$, and put $u_{\lambda}:=\mathrm{t}(\lambda) v_{\lambda}^{-1}$. Then by $[14,(2.5 .4)] \Omega=\{e\} \cup\left\{u_{\varpi_{j}^{\vee}}\right\}_{j \in J}$ with $J:=\left\{i \in 1, \ldots, N \mid\left\langle\varpi_{i}^{\vee}, \phi\right\rangle=1\right\}$. If $\lambda \in P^{\vee} \backslash Q^{\vee}$, we can write $\mathrm{t}(\lambda)=u_{\varpi_{j}^{\vee}} w$ for some $j \in J$ and $w \in W_{Q^{\vee}}$ (using $\left.W=\Omega \ltimes W_{Q^{\vee}}\right)$, and then $\mathrm{t}(\lambda)=\mathrm{t}\left(\varpi_{j}^{\vee}\right) v_{\varpi_{j}^{\vee}}^{-1} w=\mathrm{t}\left(\varpi_{j}^{\vee}\right) \mathrm{t}(\alpha) w^{\prime}$ for some $\alpha \in Q^{\vee}$ and $w^{\prime} \in W_{0}$ (using $W_{Q^{\vee}}=Q^{\vee} \rtimes W_{0}$ ). But then $w^{\prime}=e$ and $\lambda=\varpi_{j}^{\vee}+\alpha$. In particular, $\{0\} \cup\left\{\varpi_{j}^{\vee}\right\}_{j \in J}$ is a complete set of representatives of $P^{\vee} / Q^{\vee}$. Since $Q^{\vee}=\operatorname{span}_{\mathbb{Z}}\left\{w\left(\phi^{\vee}\right) \mid w \in W_{0}\right\}$, it thus suffices to show $\left(Y^{\lambda}\right)^{\circ}=T_{w_{0}} Y^{w_{0}(\lambda)} T_{w_{0}}^{-1}$ only for $\lambda=\varpi_{j}^{\vee}$ with $j \in J$ and for $\lambda=w\left(\phi^{\vee}\right)\left(w \in W_{0}\right)$.

Let $j \in J$ and write $u_{j}:=u_{\varpi_{j}^{\vee}}$ and $v_{j}:=v_{\varpi_{j}^{\vee}}$. By [14, (3.3.3)], we have $u_{j}=T_{w} Y^{w^{-1}\left(\varpi_{j}^{\vee}\right)} T_{v_{j} w}^{-1}$ for all $w \in W_{0}$. Let $\bullet: H(\underline{k}) \rightarrow H\left(\underline{k}^{-1}\right)$ denote the inverse of ${ }^{\circ}$. It follows that

$$
\begin{aligned}
\left(Y^{w_{0}\left(\varpi_{j}^{\vee}\right)}\right)^{\bullet} & =\left(T_{w_{0}}^{-1} u_{j} T_{v_{j} w_{0}}\right)^{\bullet}=T_{w_{0}} u_{j} T_{w_{0} v_{j}^{-1}}^{-1} \\
& =T_{w_{0}} u_{j}\left(T_{w_{0}} T_{v_{j}}^{-1}\right)^{-1}=T_{w_{0}} u_{j} T_{v_{j}} T_{w_{0}}^{-1} \\
& =T_{w_{0}} Y^{\varpi_{j}^{\vee}} T_{w_{0}}^{-1},
\end{aligned}
$$

since $u_{j} T_{v_{j}}=T_{u_{j} v_{j}}=T_{\mathrm{t}\left(\varpi_{j}^{\vee}\right)}=Y^{\varpi_{j}^{\vee}}$. Hence, $\left(Y^{\varpi}\right)^{\vee}=T_{w_{0}} Y^{w_{0}\left(\varpi_{j}^{\vee}\right)} T_{w_{0}}^{-1}$. Similarly, we can use $[14,(3.3 .6)]$ to obtain $\left(Y^{w\left(\phi^{\vee}\right)}\right)^{\circ}=T_{w_{0}} Y^{w_{0} w\left(\phi^{\vee}\right)} T_{w_{0}}^{-1}$ for $w \in W_{0}$, and the proof is complete.

In order to give more explicit formulas for $L_{p}^{x}$ and $L_{p}^{y}$, we need to introduce some notation. For $\lambda \in P^{\vee}$, write $W_{0, \lambda}$ for the isotropy subgroup of $\lambda$ in $W_{0}$, and $W_{0}^{\lambda}$ for a complete set of representatives of $W_{0} / W_{0, \lambda}$. We may assume that $e \in W_{0}^{\lambda}$. Let $m_{\lambda} \in \mathbb{C}[T]^{W_{0}}$ be the associated monomial symmetric function, that is, 
$m_{\lambda}(t):=\sum_{\mu \in W_{0} \lambda} t^{\mu}$. Finally, set $\Sigma^{0}(\lambda):=\Sigma(\lambda)-W_{0} \lambda$ (recall that $\Sigma(\lambda)$ is the smallest saturated subset of $P^{\vee}$ that contains $\lambda$, cf. Sect. 4.1).

Now fix $\lambda \in P_{-}^{\vee}=-P_{+}^{\vee}$. By [14, (4.4.12)], we have for $f \in \mathbb{K}$

$$
\begin{aligned}
\left(L_{m_{\lambda}}^{x} f\right)(t, \gamma)= & \sum_{w \in W_{0}^{\lambda}} \prod_{a \in S(\mathrm{t}(-\lambda))} c_{w(a), \underline{k}, q}\left(t^{-1}\right) f\left(q^{-w(\lambda)} t, \gamma\right) \\
& +\sum_{\mu \in \Sigma^{0}(\lambda)} g_{\mu}(t) f\left(q^{-\mu} t, \gamma\right)
\end{aligned}
$$

for some $g_{\mu} \in \mathcal{M}(T)$ (here we used (2.4)). In view of (6.3), one immediately obtains a similar formula for $L_{m_{\lambda}}^{y}$.

Remark 6.3 For $\lambda=w_{0}\left(\varpi_{j}^{\vee}\right)$ with $\varpi_{j}^{\vee}$ minuscule (that is, $\left\langle\varpi_{j}^{\vee}, \alpha\right\rangle \in\{0,1\}$ for all $\alpha \in R_{+}$), we have $\Sigma^{0}(\lambda)=\emptyset$, while for $\lambda=-\phi^{\vee}$ we have $\Sigma^{0}(\lambda)=\{0\}$. In both cases, one obtains an explicit formula for $L_{m_{\lambda}}^{x}$, and the resulting operators are the Macdonald $q$-difference operators [13].

We now define the following bispectral version of Macdonald's eigenvalue problem.

Definition 6.4 We define BiSP as the set of solutions $f \in \mathbb{K}$ of the following bispectral problem:

$$
\begin{aligned}
& \left(L_{p}^{x} f\right)(t, \gamma)=p\left(\gamma^{-1}\right) f(t, \gamma), \quad \forall p \in \mathbb{C}[T]^{W_{0}}, \\
& \left(L_{p}^{y} f\right)(t, \gamma)=p(t) f(t, \gamma), \quad \forall p \in \mathbb{C}[T]^{W_{0}} .
\end{aligned}
$$

Remark 6.5 Note that BiSP is a $\mathbb{W}_{0}$-invariant $\mathbb{F}$-linear subspace of $\mathbb{K}$.

\subsection{The correspondence}

Consider the linear map $\chi_{+}: H_{0} \rightarrow \mathbb{C}$ defined by $\chi_{+}\left(T_{w}\right)=k(w)$. By $\mathbb{K}$-linear extension, we obtain a $\mathbb{K}$-linear map $\chi_{+}: H_{0}^{\mathbb{K}} \rightarrow \mathbb{K}$. It gives rise to the following correspondence between SOL and BiSP.

Theorem 6.6 The $\mathbb{K}$-linear functional $\chi_{+}: H_{0}^{\mathbb{K}} \rightarrow \mathbb{K}$ restricts to an injective $\mathbb{W}_{0}$-equivariant $\mathbb{F}$-linear map

$$
\chi_{+}: \mathrm{SOL} \rightarrow \mathrm{BiSP} .
$$

The theorem follows by restricting Cherednik's correspondence mentioned in the introduction of this section (for $M$ the formal principal series module) to SOL. Indeed, if $f \in \mathrm{SOL}$, then for fixed $\gamma \in T, f(t, \gamma)$ can be viewed as a solution of qKZ for the $H$-module $M_{\gamma}$, and then by Cherednik's correspondence $\chi_{+}(f)$ satisfies the first 
system of equations of (6.6). This holds for all $\gamma \in T$. By (6.3) and the $\iota$-invariance of SOL, it then follows that

$$
\begin{aligned}
\left(L_{p}^{y} f\right)(t, \gamma) & =\left(\iota L_{p}^{x} \iota f\right)(t, \gamma)=\left(L_{p}^{x} \iota f\right)\left(\gamma^{-1}, t^{-1}\right) \\
& =p(t)(\iota f)\left(\gamma^{-1}, t^{-1}\right)=p(t) f(t, \gamma)
\end{aligned}
$$

so also the second equation of (6.6) is satisfied.

For $\mathrm{GL}_{N}$, a detailed proof can be found in [18, §6] and the arguments used there can also be applied in the present setting.

\section{Harish-Chandra series solutions}

Application of $\chi_{+}$to the basic asymptotic solution $\Phi$ leads to a meromorphic solution $\Phi_{+}$of the bispectral problem, which can be viewed as a bispectral analog of the difference Harish-Chandra solutions of the Macdonald difference equations [12]. For root systems of type $A$, Harish-Chandra series solutions were studied before in [7] and [10]. In [18, §6.4], the Harish-Chandra series solution of type $A$ was reobtained from $\Phi_{+}(t, \gamma)$, by specializing $\gamma \in T$, yielding new results on the convergence and singularities of these solutions as a consequence of corresponding results for $\Phi$. In the final subsection, we extend this to arbitrary root systems.

\subsection{Bispectral Harish-Chandra series}

As announced, we apply the map $\chi_{+}$to the basic asymptotically free solution $\Phi$ of $\mathrm{BqKZ}$ to obtain a special meromorphic solution of the bispectral problem (see [18, $\S 6.3$ ] for $\mathrm{GL}_{N}$ ).

Definition 7.1 We call $\Phi^{+}:=\chi_{+}(\Phi) \in$ BiSP the basic Harish-Chandra series solution of the bispectral problem.

Put $\Psi^{+}:=\chi_{+}(\Psi)$. Then, $\Phi^{+}=G \Psi^{+}$and as a consequence of Proposition 5.7 and Corollary 5.9, $\Psi^{+}$is analytic on $T \backslash \mathcal{S}_{+}^{-1} \times T \backslash \mathcal{S}_{+}$, and for $(t, \gamma) \in B_{\epsilon}^{-1} \times T \backslash \mathcal{S}_{+}$, we have

$$
\Psi^{+}(t, \gamma)=\sum_{\alpha \in Q_{+}^{\vee}} \Gamma_{\alpha}^{+}(\gamma) t^{-\alpha},
$$

where $\Gamma_{\alpha}^{+}:=\chi_{+}\left(\Gamma_{\alpha}\right) \in \mathcal{M}(T)$ for all $\alpha \in Q_{+}^{\vee}$. Recall that $\Gamma_{0}(\gamma)=K(\gamma) T_{w_{0}}$ for some $K \in \mathcal{M}(T)$ (see (5.19)).

Theorem 7.2 We have

$$
\Gamma_{0}^{+}(\gamma)=k\left(w_{0}\right) K(\gamma)
$$


with $K \in \mathcal{M}(T)$ given by

$$
K(\gamma)=\prod_{\alpha \in R_{+}} \frac{\left(q_{\alpha} \gamma^{\alpha^{\vee}} ; q_{\alpha}\right)_{\infty}}{\left(q_{\alpha} k_{\alpha}^{2} \gamma^{\alpha^{\vee}} ; q_{\alpha}\right)_{\infty}}
$$

where $q_{\alpha}=q^{2 /\|\alpha\|^{2}}$ for $\alpha \in R_{+}$, as before.

Proof The definition of $\chi_{+}$and the preceding remarks imply (7.1). Let $L(\gamma)$ denote the right-hand side of (7.2). Then, $L \in \mathcal{M}(T)$ is uniquely characterized by the following properties.

(i) There exists an $\epsilon>0$ such that for $\gamma \in B_{\epsilon}, L$ admits a power series expansion

$$
L(\gamma)=\sum_{\alpha \in Q_{+}^{\vee}} l_{\alpha} \gamma^{\alpha}
$$

converging normally on compacta of $B_{\epsilon}$.

(ii) $l_{0}=1$.

(iii) $L(\gamma)$ satisfies the following system of $q$-difference equations:

$$
\left(\prod_{\alpha \in R_{+}} \prod_{r=1}^{\langle\lambda, \alpha\rangle} \frac{1-q_{\alpha}^{r} \gamma^{\alpha^{\vee}}}{1-q_{\alpha}^{r} k_{\alpha}^{2} \gamma^{\alpha^{\vee}}}\right) L\left(q^{\lambda} \gamma\right)=L(\gamma), \quad \lambda \in P_{+}^{\vee}
$$

From Theorem 5.4, it follows that $K$ satisfies (i), and since $K_{0,0}=T_{w_{0}}, K$ also satisfies (ii). It thus suffices to show that $K$ solves the $q$-difference equations in (iii).

Recall that in order to show that $\Gamma_{0}(\gamma)=K(\gamma) T_{w_{0}}$ for some $K \in \mathcal{M}(T)$, we exploited the fact that $\Phi$ is a solution of the quantum KZ equation in $t$ and investigated what taking the limit $\left|t^{-\alpha_{j}^{\vee}}\right| \rightarrow 0$ had to mean for $\Gamma_{0}(\gamma)$. We are now going to exploit the fact that $\Phi^{+}$satisfies the spectral problem

$$
\left(L_{p}^{y} \Phi^{+}\right)(t, \gamma)=p(t) \Phi^{+}(t, \gamma), \quad p \in \mathbb{C}[T]^{W_{0}},
$$

and consider the limit $\left|t^{-\alpha_{i}^{\vee}}\right| \rightarrow 0$ to obtain the desired $q$-difference equations for $\Gamma_{0}^{+}$, and hence for $K$.

Fix $\lambda \in P_{-}^{\vee}$. From formula (6.5), we deduce

$$
\begin{aligned}
\left(L_{m_{\lambda}}^{y} \Phi^{+}\right)(t, \gamma)= & \sum_{w \in W_{0}^{\lambda}} \prod_{a \in S(\mathrm{t}(-\lambda))} c_{w(a), \underline{k}, q^{-1}(\gamma) \Phi^{+}\left(t, q^{w(\lambda)} \gamma\right)} \\
& +\sum_{\mu \in \Sigma^{0}(\lambda)} g_{\mu}\left(\gamma^{-1}\right) \Phi^{+}\left(t, q^{\mu} \gamma\right)
\end{aligned}
$$


with $g_{\mu} \in \mathcal{M}(T)$. Plugging in $\Phi^{+}=G \Psi^{+}$, using (5.9) and dividing both sides by $G(t, \gamma)$, the equality $\left(L_{m_{\lambda}}^{y} \Phi^{+}\right)(t, \gamma)=m_{\lambda}(t) \Phi^{+}(t, \gamma)$ gives

$$
\begin{aligned}
m_{\lambda}(t) \Psi^{+}(t, \gamma)= & \sum_{w \in W_{0}^{\lambda}} \prod_{a \in S(\mathrm{t}(-\lambda))} c_{w(a), \underline{k}}(\gamma) \delta_{\underline{k}}^{-w(\lambda)} t^{w_{0} w(\lambda)} \Psi^{+}\left(t, q^{w(\lambda)} \gamma\right) \\
& +\sum_{\mu \in \Sigma^{0}(\lambda)} g_{\mu}\left(\gamma^{-1}\right) \delta_{\underline{k}}^{-\mu} t^{w_{0}(\mu)} \Psi^{+}\left(t, q^{\mu} \gamma\right) .
\end{aligned}
$$

Now we multiply both sides by $t^{-w_{0}(\lambda)}$ and consider the limit $\left|t^{-\alpha_{j}^{\vee}}\right| \rightarrow 0$. By (7.1), this will result in a $q$-difference equation for $K$. Note that:

(1) $t^{-w_{0}(\lambda)} m_{\lambda}(t)=\sum_{\mu \in W_{0} \lambda} t^{-w_{0}(\lambda)+\mu} \rightarrow 1$ since $w_{0}(\lambda) \in P_{+}^{\vee}$ and $v-w(v) \in Q_{+}^{\vee}$ for all $v \in P_{+}^{\vee}$ and $w \in W_{0}$.

(2) $t^{-w_{0}(\lambda)} t^{w_{0} w(\lambda)}=t^{-w_{0}(\lambda)+w_{0} w(\lambda)}$ which is equal to 1 if $w(\lambda)=\lambda$ and tends to 0 otherwise. Considering $w \in W_{0}^{\lambda}$, we have $w(\lambda)=\lambda$ only for $w=e$.

(3) $t^{-w_{0}(\lambda)} t^{w_{0}(\mu)} \rightarrow 0$ for all $\mu \in \Sigma^{0}(\lambda)$. Indeed, by [14, (2.6.3)] we have

$$
\mu_{+} \in \Sigma\left(w_{0}(\lambda)\right) \Leftrightarrow w_{0}(\lambda)-\mu_{+} \in Q_{+}^{\vee}
$$

and hence also $w_{0}(\lambda)-w_{0}(\mu) \in Q_{+}^{\vee}$ for $\mu \in \Sigma^{0}(\lambda) \subset \Sigma\left(w_{0}(\lambda)\right)$. Moreover, $w_{0}(\lambda) \neq w_{0}(\mu)$ since $\mu \notin W_{0} \lambda$.

Consequently, $K$ satisfies the following set of $q$-difference equations:

$$
\left(\prod_{a \in S(\mathrm{t}(-\lambda))} c_{a ; \underline{k}, q^{-1}}(\gamma)\right) \delta_{\underline{k}}^{-\lambda} K\left(q^{\lambda} \gamma\right)=K(\gamma), \quad \lambda \in P_{-}^{\vee} .
$$

Equivalently, also setting $\mu:=-\lambda \in P_{+}^{\vee}$,

$$
\left(\prod_{a \in S(\mathrm{t}(\mu))} \frac{k_{a}^{-1}-k_{a}\left(q^{\mu} \gamma\right)^{a^{\vee}}}{1-\left(q^{\mu} \gamma\right)^{a^{\vee}}}\right) \delta_{\underline{k}}^{\mu} K(\gamma)=K\left(q^{\mu} \gamma\right), \quad \mu \in P_{+}^{\vee}
$$

Note that $L_{m_{\lambda}}^{y} \in \mathbb{C}(T) \#_{q^{-1}} W \simeq \mathbb{C}(\{1\} \times T) \#(\{e\} \times W)$, so $\gamma^{(\alpha+r c)^{\vee}}=q_{\alpha}^{-r} \gamma^{\alpha^{\vee}}$ for $\alpha \in R$ and $r \in \mathbb{Z}$. Using

$$
\begin{aligned}
\prod_{a \in S(\mathrm{t}(\mu))} \frac{k_{a}^{-1}-k_{a}\left(q^{\mu} \gamma\right)^{a^{\vee}}}{1-\left(q^{\mu} \gamma\right)^{a^{\vee}}} & =\prod_{\alpha \in R_{+}} \prod_{r=0}^{\langle\mu, \alpha\rangle-1} \frac{k_{\alpha}^{-1}-k_{\alpha} q_{\alpha}^{\langle\mu, \alpha\rangle} q_{\alpha}^{-r} \gamma^{\alpha^{\vee}}}{1-q_{\alpha}^{\langle\mu, \alpha\rangle} q_{\alpha}^{-r} \gamma^{\alpha^{\vee}}} \\
& =\prod_{\alpha \in R_{+}} \prod_{r=1}^{\langle\mu, \alpha\rangle} \frac{k_{\alpha}^{-1}-k_{\alpha} q_{\alpha}^{r} \gamma^{\alpha^{\vee}}}{1-q_{\alpha}^{r} \gamma^{\alpha^{\vee}}}
\end{aligned}
$$


and $\delta_{\underline{k}}^{\mu}=\prod_{\alpha \in R_{+}} k_{\alpha}^{\langle\mu, \alpha\rangle}$, we obtain from (7.4)

$$
\left(\prod_{\alpha \in R_{+}} \prod_{r=1}^{\langle\mu, \alpha\rangle} \frac{1-k_{\alpha}^{2} q_{\alpha}^{r} \gamma^{\alpha^{\vee}}}{1-q_{\alpha}^{r} \gamma^{\alpha^{\vee}}}\right) K(\gamma)=K\left(q^{\mu} \gamma\right), \quad \mu \in P_{+}^{\vee},
$$

and the proof is complete.

In view of Remark 6.5, we obtain solutions $\Phi_{w}^{+} \in \operatorname{BiSP}\left(w \in W_{0}\right)$, given by

$$
\Phi_{w}^{+}(t, \gamma):=\Phi^{+}\left(t, w^{-1} \gamma\right)
$$

Setting $\Psi_{w}^{+}(t, \gamma):=\Psi^{+}\left(t, w^{-1} \gamma\right)$, we have $\Phi_{w}^{+}(t, \gamma)=G\left(t, w^{-1} \gamma\right) \Psi_{w}^{+}(t, \gamma)$ and by Corollory 5.11(ii), for $\epsilon>0$ sufficiently small, $\Psi_{w}^{+}$has a power series expansion

$$
\Psi_{w}^{+}(t, \gamma)=\sum_{\alpha \in Q_{+}^{\vee}} \Gamma_{\alpha}^{+}\left(w^{-1} \gamma\right) t^{-\alpha}
$$

for $(t, \gamma) \in B_{\epsilon} \times T \backslash w\left(\mathcal{S}_{+}\right)$, converging normally on compacta of $B_{\epsilon} \times T \backslash w\left(\mathcal{S}_{+}\right)$. The next result follows along the same line as [18, Prop. 6.20].

Proposition 7.3 The set $\left\{\Phi_{w}^{+}\right\}_{w \in W_{0}} \subset \mathrm{BiSP}$ is $\mathbb{F}$-linearly independent.

We expect that the set $\left\{\Phi_{w}^{+}\right\}_{w \in W_{0}}$ is in fact a basis of BiSP over $\mathbb{F}$. This would follow, for example, if we could prove that $\chi_{+}$is an $\mathbb{F}$-linear isomorphism SOL $\rightarrow$ BiSP (rather than only an embedding). Both are still open problems.

\subsection{Application to Harish-Chandra series solutions} of Macdonald's difference equations

Let $\zeta \in T$. The spectral problem of the Macdonald $q$-difference operators with spectral parameter $\zeta$ is

$$
L_{p}^{x} f=p\left(\zeta^{-1}\right) f, \quad \forall p \in \mathbb{C}[T]^{W_{0}},
$$

for meromorphic functions $f$ on $T$. Let $\mathrm{SP}_{\zeta} \subset \mathcal{M}(T)$ denote the set of solutions of (7.5). It is a vector space over $\mathcal{E}(T)$, invariant under the usual action of $W_{0}$ on $M(T)$.

Recall the solution space $\mathrm{SOL}_{\zeta} \subset H_{0}^{\mathcal{M}(T)}$ of the quantum KZ equation (3.11) associated with $M_{\zeta}$, also $W_{0}$-invariant, but with respect to the $\tau_{x}^{M_{\zeta}}\left(W_{0}\right)$-action on $H_{0}^{\mathcal{M}(T)}$. We have the following special case of Cherednik's correspondence from [2,3] (see [18, Prop. 6.22]).

Proposition 7.4 For each $\zeta \in T, \chi_{+}$defines an $W_{0}$-equivariant $\mathcal{E}(T)$-linear map $\chi_{+}: \mathrm{SOL}_{\zeta} \rightarrow \mathrm{SP}_{\zeta}$

Remark 7.5 In Stokman's paper [15], it is shown that $\chi_{+}$is an isomorphism if $\zeta^{\alpha^{\vee}} \neq$ $k_{\alpha}^{2}, 1$ for all $\alpha \in R$. 
Recall that $\Psi^{+}=\chi_{+}(\Psi)$ with $\Psi$, as usual, the solution of the gauged bispectral BqKZ Eqs. (5.11) obtained in Theorem (5.4). It follows from Corollary 5.11 that $\Psi^{+}(t, \gamma)$ may be specialized at $\gamma=\zeta$ for $\zeta \in T \backslash \mathcal{S}_{+}^{-1}$, yielding a meromorphic function $\Psi^{+}(\cdot, \zeta) \in \mathcal{M}(T)$ with poles at $t \in \mathcal{S}_{+}^{-1}$. Define $\widetilde{G} \in \mathbb{K}$ by

$$
\widetilde{G}(t, \gamma):=\frac{\theta_{q}\left(t w_{0}(\gamma)^{-1}\right)}{\theta_{q}\left(\delta_{\underline{k}} t\right)} .
$$

Remark 7.6 Note that $\widetilde{G}(t, \gamma)=\theta_{q}\left(\delta_{\underline{k}}^{-1} w_{0}(\gamma)^{-1}\right) G(t, \gamma)$ and that $\widetilde{G}(t, \gamma)$ can be specialized in $\gamma=\zeta$. Lacking the factor $\theta_{q}\left(\delta_{\underline{k}}^{-1} w_{0}(\gamma)^{-1}\right)$ in the denominator, $\widetilde{G}$ does not satisfy $\iota(\widetilde{G})=\widetilde{G}$. Therefore, $\widetilde{G} \Psi \notin \operatorname{SOL}$, but we do have $\widetilde{G}(\cdot, \zeta) \Psi(\cdot, \zeta) \in \operatorname{SOL}_{\zeta}$.

It follows that $\widetilde{G}(\cdot, \zeta) \Psi^{+}(\cdot, \zeta) \in \mathrm{SP}_{\zeta}$ and hence $\Psi^{+}(\cdot, \zeta)$ is a solution of the spectral problem for the gauged Macdonald $q$-difference operators with spectral parameter $\zeta$, that is, a solution of

$$
\left(\widetilde{L}_{p}^{x} f\right)(t)=p\left(\zeta^{-1}\right) f(t), \quad \forall p \in \mathbb{C}[T]^{W_{0}},
$$

with

$$
\widetilde{L}_{p}^{x}:=\widetilde{G}(\cdot, \zeta)^{-1} L_{p}^{x} \widetilde{G}(\cdot, \zeta)
$$

At the end of the previous subsection, we introduced $\Psi_{w}^{+}(t, \gamma)=\Psi^{+}\left(t, w^{-1} \gamma\right)$ for $w \in W_{0}$. Put $\mathcal{S}:=\bigcup_{w \in W_{0}} w\left(\mathcal{S}_{+}\right)$. The considerations of this section imply the following.

Theorem 7.7 Fix $\zeta \in T \backslash S$.

(i) For $\epsilon>0$ sufficiently small, $\Psi_{w}^{+}(\cdot, \zeta)$ has a power series expansion

$$
\Psi_{w}^{+}(t, \zeta)=\sum_{\alpha \in Q_{+}^{\vee}} \Gamma_{\alpha}^{+}\left(w^{-1} \zeta\right) t^{-\alpha}
$$

for $t \in B_{\epsilon}$, converging normally on compacta of $B_{\epsilon}$ and with $\Gamma_{0}^{+}\left(w^{-1} \zeta\right) \neq 0$ explicitly given by (7.1).

(ii) $\Psi_{w}^{+}(t, \zeta)\left(w \in W_{0}\right)$ is analytic in $t \in T \backslash \mathcal{S}_{+}^{-1}$.

(iii) The function $\widetilde{\Psi}_{w}^{+}(\cdot, \zeta) \in \mathcal{M}(T)\left(w \in W_{0}\right)$ defined by

$$
\widetilde{\Psi}_{w}^{+}(t, \zeta):=\frac{\widetilde{G}\left(t, w^{-1}(\zeta)\right)}{\widetilde{G}(t, \zeta)} \Psi_{w}^{+}(t, \zeta)=\frac{\theta_{q}\left(t\left(w_{0} w^{-1}\right)(\zeta)^{-1}\right)}{\theta_{q}\left(t w_{0}(\zeta)^{-1}\right)} \Psi_{w}^{+}(t, \zeta),
$$

is a nonzero solution of the spectral problem (7.6) for the gauged Macdonald $q$-difference operators for all $w \in W_{0}$.

The functions $\widetilde{\Psi}_{w}^{+}(\cdot, \zeta)\left(w \in W_{0}\right)$ are the Harish-Chandra series solutions of the spectral problem (7.6). As already mentioned in the introduction of this section, formal Harish-Chandra series solutions of Macdonald's spectral problem were already 
obtained in [12], and earlier for the root system of type $A$ in [7] and [10]. The upshot here is that we obtain the Harish-Chandra series solutions as meromorphic functions and are able to explicitly determine the leading term and the pole locations of $\Psi_{w}^{+}(\cdot, \zeta)$.

Acknowledgments The author is supported by the Netherlands Organization for Scientific Research (NWO) in the VIDI-project "Symmetry and modularity in exactly solvable models". He likes to thank Jasper Stokman for his advice and many valuable discussions.

Open Access This article is distributed under the terms of the Creative Commons Attribution Noncommercial License which permits any noncommercial use, distribution, and reproduction in any medium, provided the original author(s) and source are credited.

\section{References}

1. Cherednik, I.: Quantum Knizhnik-Zamolodchikov equations and affine root systems. Commun. Math. Phys. 150, 109-136 (1992)

2. Cherednik, I.: Double affine Hecke algebras, Knizhnik-Zamolodchikov equations, and Macdonalds operators. Int. Math. Res. Not. 9, 171-180 (1992)

3. Cherednik, I.: Induced representations of double affine Hecke algebras and applications. Math. Res. Lett. 1, 319-337 (1994)

4. Cherednik, I.: Double affine Hecke algebras and difference Fourier transforms. Invent. Math. 152(2), 213-303 (2003)

5. Cherednik, I.: Double Affine Hecke Algebras, London Mathematical Society Lecture Note Series, vol. 319. Cambridge University Press, Cambridge (2005)

6. Etingof, P.I.: Difference Equations with Elliptic Coefficients and Quantum Affine Algebras. preprint (1993), arXiv:hep-th/9312057

7. Etingof, P.I., Kirillov, A.A. Jr..: Macdonald's polynomials and representations of quantum groups. Math. Res. Lett. 1(3), 279-296 (1994)

8. Etingof, P., Varchenko, A.: Dynamical Weyl groups and applications. Adv. Math. 167(1), 74-127 (2002)

9. Kato, S.: $R$-matric arising from affine Hecke algebras and its applications to Macdonald's difference operators. Commun. Math. Phys. 165(3), 533-553 (1994)

10. Kazarnovski-Krol, A.: Matrix elements of vertes operators of the deformed $\mathrm{WA}_{n}$-algebra and the Harish-Chandra solutions to Macdonald's difference equations. Sel. Math. New Ser. 5(2), 257301 (1999)

11. Looijenga, E.: Root systems and elliptic curves. Invent. Math. 38, 17-32 (1976)

12. Letzter, G., Stokman, J.V.: Macdonald difference operators and Harish-Chandra series. Proc. Lond. Math. Soc. 97(1), 60-96 (2008)

13. Macdonald, I.G.: Orthogonal Polynomials Associated with Root Systems, preprint (1987). Séminaire Lotharingien Combin. 45, 1-40 (2000)

14. Macdonald, I.G.: Affine Hecke Algebras and Orthogonal Polynomials. Cambridge Tracts in Mathematics, vol. 157. Cambridge University Press, Cambridge (2003)

15. Stokman, J.V.: Quantum affine Knizhnik-Zamolodchikov equations and quantum spherical functions, I, Int. Math. Res. Not. doi:10.1093/imrn/rnq094

16. Stokman, J.V.: Quantum affine Knizhnik-Zamolodchikov equations and quantum spherical functions, II (in preparation)

17. Takeyama, Y.: Differential equations compatible with boundary rational qKZ equation, preprint (2009), arXiv:0908.2288

18. van Meer, M., Stokman, J.V.: Double affine Hecke algebras and bispectral quantum KnizhnikZamolodchikov equations, preprint (2008), Int. Math. Res. Not. 2010(6), 969-1040 (2010) 